\title{
SIGN LUMINANCE REQUIREMENTS FOR VARIOUS BACKGROUND COMPLEXITIES
}

L...

DO

Research, Development and Technology

Turner-Fairbank Highway

Research Center

6300 Georgetown Pike

McLean, Virginia 22101

Report No.

FHWA/RD-85/056

U.S. Department

of Transportation

Final Report

Federal Highway

September 1985

Administration

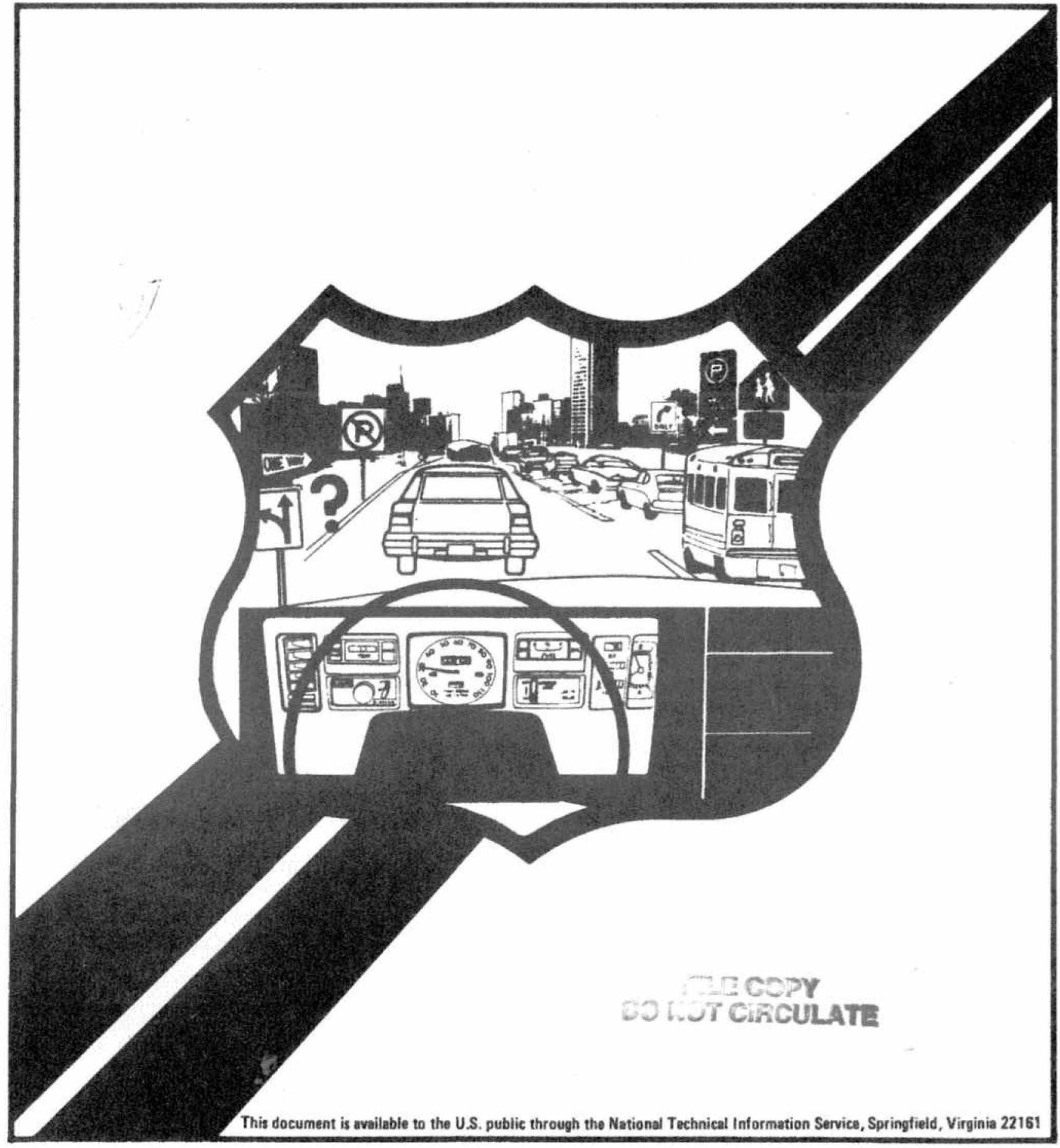




\section{FOREWORD}

This research report is primarily intended for use by traffic engineers in determining what retroreflective material will provide adequate sign detection in a given location. The study will also be of interest to those traffic operations engineers who are concerned with when signs have reached their useful end of life. The study was initiated at the request of the Federal Highway Administration's (FHWA) Office of Traffic Operations and the National Committee on Uniform Traffic Control Devices.

The report describes research aimed at determining whether a particular warning sign has a complex background and what range of sign luminances are adequate for detection of that sign under normal nighttime conditions. A procedure was developed involving field rating of potential sign locations on four subjective scales to distinguish low visual complexity background scenes from high complexity scenes.

Sufficient copies of the report are being distributed to provide single copies to each FHWA regional office. Copies are also being distributed to the National Committee on Uniform Traffic Control Devices' Technical Committee on Signs and the American Association of State Highway Transportation Officials' Traffic Engineering Subcommittee.

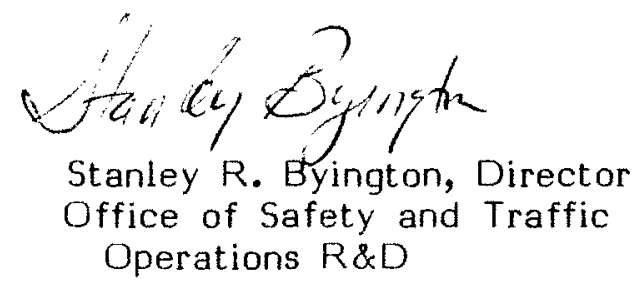

\section{NOTICE}

This document is disseminated under the sponsorship of the Department of Transportation in the interest of information exchange. The United States Government assumes no liability for its content or use thereof.

The contents of this report reflect the views of the contractor, who is responsible for the facts and the accuracy of the data presented herein. The contents do not necessarily reflect the official policy of the Departinent of Transportation.

This report does not constitute a standard, specification, or regulation.

The United States Government does not endorse products or manufacturers. Trademarks or manufacturers' names appear herein only because they are considered essential to the object of this document. 


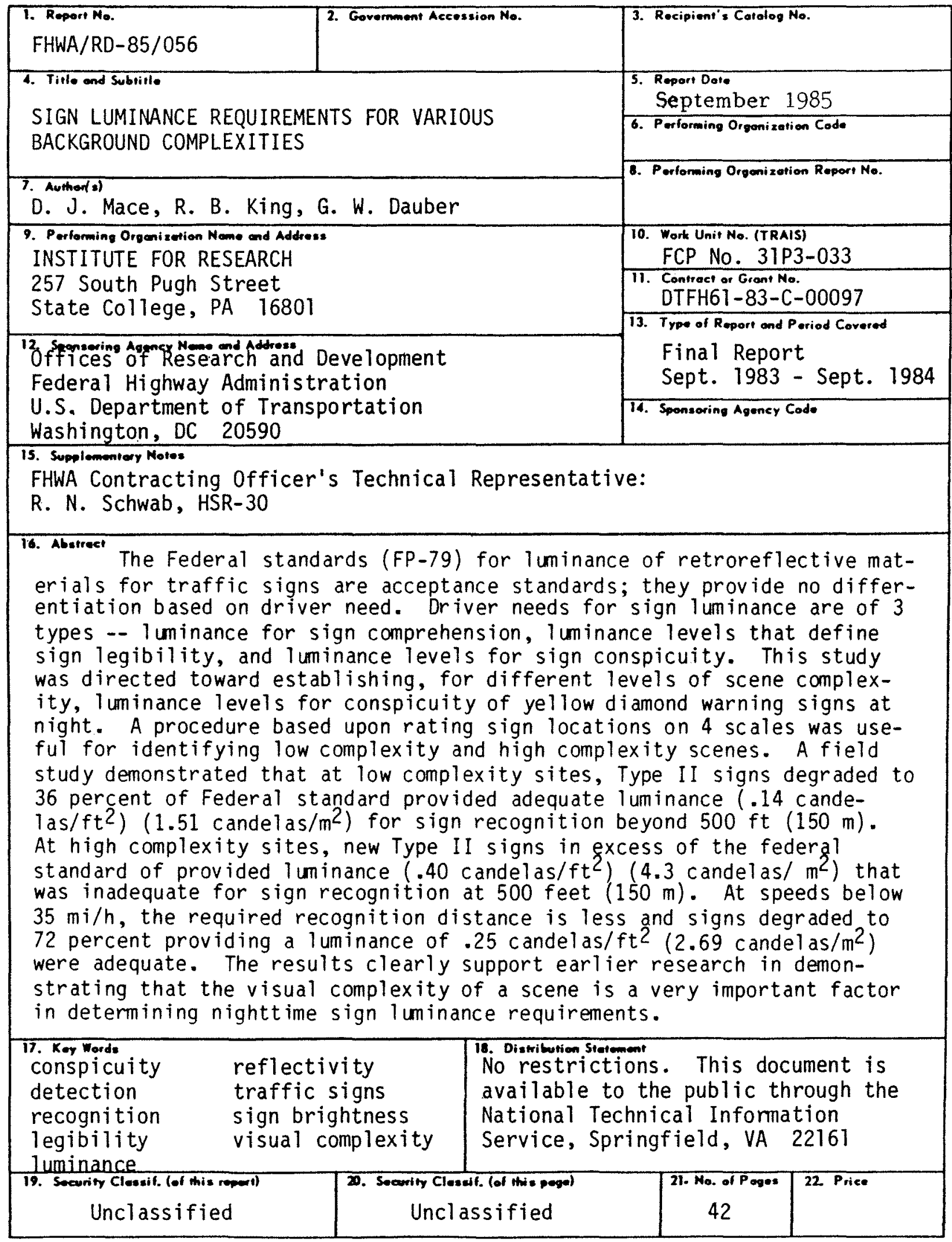

\footnotetext{
Form DOT F 1700.7 (8-72) Roproduction of completed poge outhorized
}

Federal Highway Admin. Technical Roference Center 6300 Coorgetown Pike Milean, VA $22101-2296$ 
The authors thank agencies that contributed services and materials to this research. They include the New Jersey Department of Transportation, which provided vehicles and facilities for administering tests and personnel to serve as subjects, the Pennsylvania Department of Transportation, which lent a retroreflectometer for verifying test sign degradation levels, and 3-M Company, which provided materials for producing degraded retroreflective test signs. 
LIST OF TABLES • • • • • • • • • • • • • • • • • • • • • • • • • • • • • • • • IV

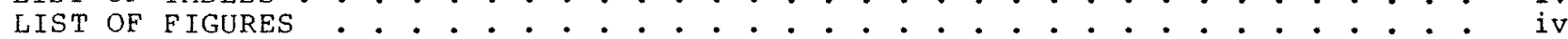

I. EXECUTIVE SUMMARY . . . . . . . . . . . . . . . . . . . . . . . . . 1

II. BACKGROUND . . . . . . . . . . . . . . . . . . . . . . . . . . . . 2

A. Luminance Standards and Driver Needs . . . . . . . . . . . . . . . . 2

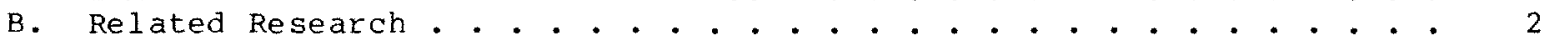

C. Purpose . . . . . . . . . . . . . . . . . . . . . . . . . . . . 4

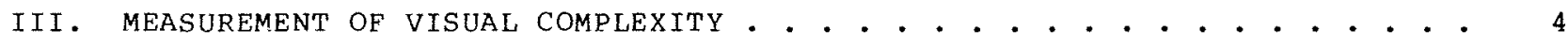

A. Approaches to the Measurement of Complexity . . . . . . . . . . . . 4

B. Development of Complexity Scales...... . . . . . . . . . . . . . 5

IV. Field VALIDATION OF VISUAL COMPLEXITY . . . . . . . . . . . . . . . . . . . 7

A. General Experimental procedure . . . . . . . . . . . . . . . . 7

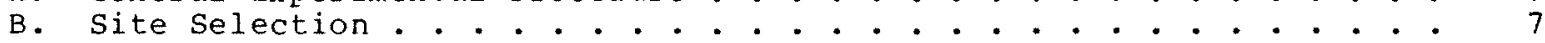

C. Phase I: The Measurement of Complexity . . . . . . . . . . . . . . 9

Site Preparation . . . . . . . . . . . . . . . . . . . . . . 9

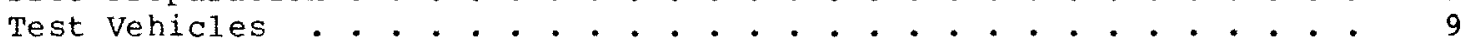

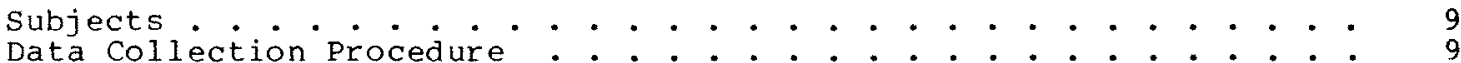

D. Phase II: The Acquisition of Sign Recognition and

Legibility Distances . . . . . . . . . . . . . . . . . . . . 12

Site preparation . . . . . . . . . . . . . . . . . . . . . 12

Variables . . . . . . . . . . . . . . . . . . . . . . . . . 12

Test Vehicles . . . . . . . . . . . . . . . . . . . . . . . . . . 12

Subjects . . . . . . . . . . . . . . . . . . . . . . . . . 12

Experimental Design . . . . . . . . . . . . . . . . . . . . . . . . . . 12

Data Collection Procedure . . . . . . . . . . . . . . . . . . . . . . . 13

E. Results . . . . . . . . . . . . . . . . . . . . . . . 13

Analysis of Complexity Scales . . . . . . . . . . . . . . . . . . . 13

Analysis of Recognition/Legibility Data . . . . . . . . . . . . . . 15

Decision Rules for Luminance Requirements . . . . . . . . . . . . 18

F. Conclusions . . . . . . . . . . . . . . . . . . . . . . . 22

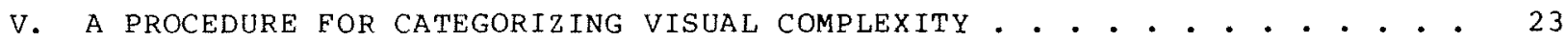

APPENDIX A - FACTOR ANALYSTS PROCEDURE AND RESUlTS . . . . • • . . . . . . . 28

APPENDIX B - INSTRUCTIONS FOR COMPLEXITY EVALUATION (FIELD STUDY) • • • . • . 28

Purpose of the study . . . . . . . . . . . . . . . . . . . . . 28

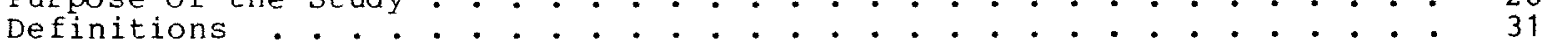

Complexity Evaluation . . . . . . . . . . . . . . . . . . . . . . 31

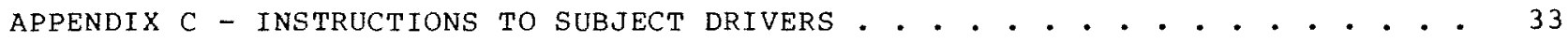

APPENDIX D - SUGGESTED REVISION TO TRAFEIC CONTROL DEVICES HANDBOOK • • • • • 34

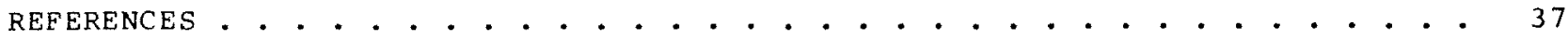


1 Site characteristics . . . . . . . . . . . . . . . . . . . 10

2 Spearman-Brown reliability coefficient for each scale and 2 groups of raters ..................................... 13

3 Zero-order correlations of 8 complexity scales with performance

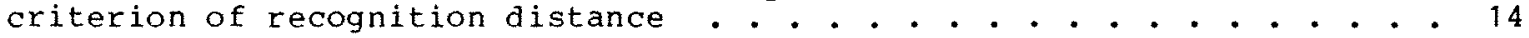

$4 \mathrm{R}^{2}$ using varying numbers of scales from photo 2 and road groups . . . . 14

5 Recognition distance ( $f t$ ) performance by site and sign brightness

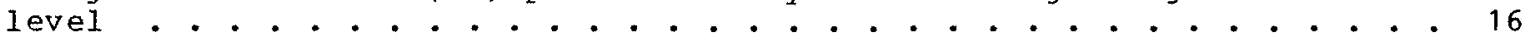

6 Mean legibility distance (ft) by site and sign brightness level . . . 16

7 Predicted versus actual recognition distances (ft) (12 sites) . . . . 15

8 Observed mean recognition distances (ft) . . . . . . . . . . . . 17

9 Observed mean legibility distances (ft) . . . . . . . . . . . . . . 17

10 Required recognition and legibility distances (ft) . . . . . . . . . . 18

11 Evaluation of 3 cutoff scores for identifying 8 low complexity sites

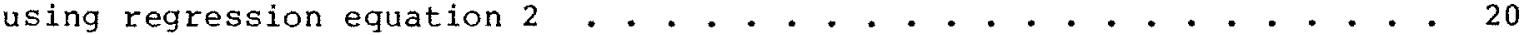

12 Evaluation of 3 cutoff scores for identifying 8 low complexity sites

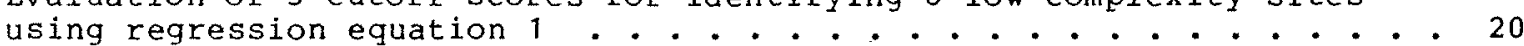

13 Evaluation of 3 cutof scores for identifying 8 high complexity sites using regression equation 2 . . . . . . . . . . . . . . . . . . . . . . 21

14 Evaluation of 3 cutoff scores for identifying 8 high complexity sites

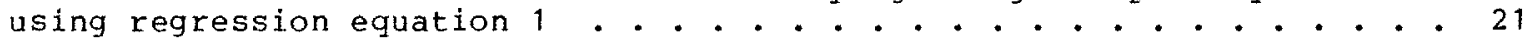

15 Variable factor weights . . . . . . . . . . . . . . . . . 29

LIST OF FIGURES

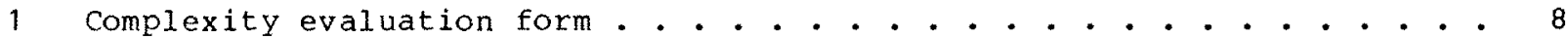

2 Recognition performance by visual complexity and sign brightness . • • 15

3 Legibility performance by visual complexity and sign brightness . • • • 17

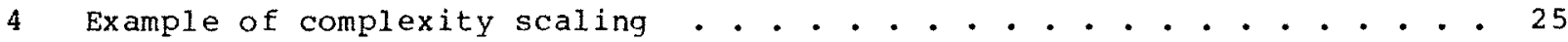

5 Visual complexity rating form . . . . . . . . . . . . . . . . . . 26 


\section{EXECUTIVE SUMMARY}

The choice among retroreflective sheeting materials that conform to Federal standards (FP-79) involves economics and safety. Type III sheeting (high intensity) offers longer service life with greater legibility and conspicuity at considerably higher cost than Type II sheeting (engineering grade). Wherever increased service life or added conspicuity is needed, Type III sheeting may be worth its additional cost. At many sign locations, however, signs are replaced (because of vandal ism or accident) at a frequency where service life is not a factor. Also, many sites with little visual complexity do not require excess luminance to satisfy nighttime driver requirements.

The research reported here considered the role of sign brightness and the visual complexity of the highway environment in the recognizability and legibility of traffic signs at a variety of nighttime field situations. The purpose was to advance earlier work in this area by developing a practical procedure for categorizing the visual complexity of highway scenes and to determine reasonable luminance standards for yellow warning signs for different levels of background complexity.

Factor analysis techniques were applied to a group of 40 visual variables descriptive of highway scenes which were identified in a previous study. Eleven dimensions with both theoretical and practical meaning were selected and scaled for the subjective rating of visual complexity. The scales were first evaluated using photographic projections of 80 scenes from the earlier study for which sign recognition criteria were available. The 11 scales were reduced to 8 and used to rate the complexity of 24 new sites along a $24-\mathrm{mi}(38.4-\mathrm{km})$ loop near Trenton, New Jersey. Subjects were divided into 2 groups to allow comparison of a photographic rating procedure and an on-site field rating procedure. The onsite procedure provided far more reliable ratings.

A field study was conducted to estab1 ish the validity of the rating scales and to determine the luminance requirements of drivers for sign recognition and legibility as a function of the scaled visual complexity. Three brightness levels of yellow diamond warning signs were installed at the Trenton test sites, and individual sign recognition and legibility distances were recorded for each of 15 subject drivers who drove the 24$\mathrm{mi}(38.4-\mathrm{km})$ 10op.
Multiple regression techniques were used to produce a simple equation using 4 scales which had a high degree of accuracy in discriminating between sites with long and short sign recognition distances. The procedure for using these 4 scales for classifying sign locations with respect to visual complexity is explained in chapter $V$.

Data analysis indicated that sign brightness improved both recognition and legibility distances, but that visual complexity had no effect on legibility. The obtained recognition and legibility distances were examined relative to requirements predicted from a driver decision model. This comparison revealed that at low complexity sites, signs which had deqraded below Federal acceptance standards for accelerated weathering provided recognition distances well in excess of predicted requirements. At high complexity locations, signs with retro-reflection greater than the Federal specification for Type II sheeting (engineering grade) sometimes had recognition distances less than predicted requirements. The results suggest that Type III sheeting (high intensity), or larger signs or advance warning signs, may be needed at high complexity locations, particularly where speed limits are high and speed reduction or lane-change maneuvers are reguired.

The predicted requirements on which these conclusions are based vary with the approach speed and the type of maneuver required, e.g., deceleration to $35 \mathrm{mi} / \mathrm{h}$ $(56 \mathrm{~km} / \mathrm{h})$. A single standard for all yellow warning signs must provide for the greatest recognition requirements and this was done in developing these general conclusions.

When visual complexity of a shoulder or right side sign location is low, yellow warning signs with Type II sheeting could be allowed to degrade to an SIA of 18 (36 percent of Federal specification for new material). However, when visual complexity is high, such signs should be replaced if their retro-reflection degrades to an SIA of 36 ( 72 percent of Federal specification for new material). When approach speeds are in excess of $45 \mathrm{mi} / \mathrm{h}$ $(72 \mathrm{~km} / \mathrm{h})$, or if alignment will decrease headlight illumination, even new Type II sheeting may not be adequate, and either Type III sheeting or advance warning signs should be considered. 


\section{BACKGROUND}

A. Luminance Standards and Driver Needs

The Federal standards for the luminance of retroreflective materials for traffic signs are acceptance standards (U.S. Department of Transportation, 1979, section 633). These standards, measured as specific intensity per unit area (SIA), provide absolute minimum requirements for different materials and colors, but provide no differentiation based upon driver need.

Driver needs for sign luminance are of 2 types -- luminance requirements for legibility and luminance requirements for conspicuity. These requirements are separate and distinct. In general, a sign must first get the driver's attention and then provide sufficient legibility to effectively transmit its information. While conspicuity is less important for signs following advance warning signs, it may still be needed in situations where the first sign is not perceived. If both signs are inconspicuous the effectiveness of their information redundancy is significantly reduced.

There are at least 2 reasons why Federal luminance standards are not responsive to either of these driver requirements. First, the vagaries of behavioral research and the variance in human behavior are such that precision in specifying driver requirements is difficult to achieve. Second, it is very difficult if not impossible to precisely measure luminance in the field, so there is yet no practical method of determining whether the requirements were met if they were established. The large variance in driver requirements coupled with the large variance in luminance or other sign performance measures would make any decision criteria suspect.

\section{B. Related Research}

Two research groups that have recently studied sign conspicuity call attention to the importance of background complexity in the study of conspicuity:

Jenkins (Note 1) stated, "No object is conspicuous per se. It can only be conspicuous in a certain background; if the background changes then the object may or may not remain conspicuous."

Mace, Perchonok, and Pollack (1982) expanded this observation, giving equal importance to the role of driver motivation and uncertainty: conspicuity, like visibility and legibility, is not an observable characteristic of a sign, but a construct that relates measures of perceptual performance with measures of background, motivation, and driver uncertainty.

A STOP sign following a STOP AHEAD sign will be more conspicuous because the driver's uncertainty about its presence is reduced. Directional signs are more easily recognized by drivers looking for them, and the increased motivation which occurs when someone is lost makes all signs more conspicuous. In studying conspicuity, we believe that levels of uncertainty and motivation (which are independent driver characteristics) should be established as part of the design driver concept.

Cole and Jenkins (1980) define a conspicuous object as one that will, for a given background, be seen with "certainty" (greater than 90 percent probability of detection) within a short observation time ( $250 \mathrm{~ms}$ ), regardless of its location in relation to the line of fixation. They found that size had a more significant effect on detection in daytime highway scenes than target contrast, but that the complexity of the scene had a major effect as well.

The most abundant literature relevant to sign conspicuity would probably be in the area of visual noise and target/surround relationships. While this literature offers many basic principles of target detection which should be considered when measuring the complexity of highway scenes, most of these principles have little practical relevance to the problem of sign recognition. A review of these studies was provided by Mace and Pollack (1983). The principles they found follow.

The recognition of a target, such as a traffic sign, is influenced by the characteristics of the target and by the contrast of these target characteristics with similar dimensions of the surround. For example, the attention-getting value of a target increases as:

- The target's brightness increases (Forbes, Fry, Joyce, \& Pain, 1968; Monk, 1976).

- The brightness contrast between the target and its surround increases (Forbes et al., 1968; Forbes, Pain, Fry, \& Joyce, 1967; Forbes, Pain, Joyce, \& Fry, 1968; Hanson \& Dickson, 1963; Lovie \& Lovie, 1970; Pain, $1969)$.

- The brightness contrast between different parts of the target increases (e.g., sign legend to background) 
(Forbes, Fry, Joyce, \& Pain, 1968; Forbes, Pain, Joyce, \& Fry, 1968).

- The target's size increases relative to other stimuli in the visual field (Bloomfield, 1972; Forbes, Fry, Joyce, \& Pain, 1968; Steedman \& Baker, 1960).

- The shape of the target contrasts with noise items (Dornic \& Borg, 1971).

- The target's hue contrasts with noise (Forbes, Pain, Joyce, \& Fry, 1968; Saenz \& Riche, 1974).

In addition to the effects of target characteristics, the characteristics of a target's surround also influence the likelihood of target detection. Specifically, several basic studies suggest that target conspicuity increases as:

- The number of noise elements in the $v$ isual field decreases (Baker, Morris, \& Steedman, 1960; Banks, Bodinger, \& I11ige, 1974; Brown and Monk, 1975; Cahill \& Carter, 1976; Crawford, 1962; Eriksen \& Eriksen, 1974; McIntyre, Fox, \& Neale, 1970).

- The overall density of noise items in the visual field decreases (Green \& Anderson, 1956; Smith, 1962; Smith \& Thomas, 1964).

- The density of noise items immediately adjacent to the target decreases (Monk \& Brown, 1975).

- The distance between the target and noise increases (Banks, Bachrack, \& Larson, 1977; Banks, Bodinger, \& Illige, 1974; Brown \& Monk, 1975; Eriksen \& Eriksen, 1974).

- The target is located further from the center of the visual field than the noise (versus when the target is located closer to the center of the visual field than the noise) (Banks, Bachrack, \& Larson, 1977; Estes \& Wolford, 1971; Mackworth, 1965; Shaw, 1969; Wolford \& Hollingsworth, 1974).

- The number of irrelevant classes of stimuli in the visual field decreases (i.e., as the visual field becomes more homogeneous) (Eriksen, 1953).
- The variability within each irrelevant class of stimuli decreases (Smith \& Thomas, 1964).

Because the majority of studies in this area reflect basic research that often uses abstract targets located within relatively sterile visual matrices, operational definitions for measurement of complexity in highway scenes have not been established.

In an attempt to operationally define visual complexity as it relates to the nighttime conspicuity of retroreflective traffic signs, Mace et al. (1982) conducted an extensive laboratory study with 80 different highway scenes and 4 types of traffic signs. Approximately 40 different visual and photometric measures were made of scene, target, and surround.

Because the role of visual complexity in detection and recognition is not well understood, target and contrast variables were included so that their relative importance and interactions could be evaluated. The performance criterion was the proportion of correct sign recognitions. ${ }^{1}$ A correct response required identifying the type of sign present in a scene (i.e., STOP, rectangular orange, black on white regulatory, or yellow diamond warning).

This study resulted in a number of interesting findings:

- When visual complexity of the scene is high, complexity is a more significant determinant of sign recognition than contrast of the sign to its surround. (When visual complexity is low, conspicuity is not an issue and target contrast and size would determine detection.)

- Black on white regulatory signs have poorer conspicuity than other signs, even at close distances.

- Yellow diamond warning signs have greater conspiciuty than other signs at long distances.

- Increasing the brightness of signs (except black on white) can offset the decrease in conspicuity from increased visual complexity. (Increasing the brightness of the black on white regulatory sign may not improve its conspicuity.)

\footnotetext{
1 It is the authors' contention that because of the complexity and number of targets along the highway, conspicuity cannot be studied using a target less well-defined. The basic threshold paradigms, "Tell me when you see something" or "Tell me when the sign is visible," are not applicable. The first paradigm won't work because there is too much out there to report seeing. The second paradigm is not appropriate because it reguires that you reduce the uncertainty about target type and location so that threshold perception and not conspicuity is measured.
} 
A small field study at only 3 sites suggested that, at the extremes of visual complexity, sign brightness (within the typical range of retroreflective luminances) might not affect recognition or conspicuity. It was suggested that at high levels of visual complexity, the highest available retroreflective luminance might not provide adequate recognition distance; while at very low levels of visual complexity, all signs, even those degraded to the Federal standards for accelerated weathering (U.S. Department of Transportation, 1979, section 633 ), would satisfy driver requirements.

While the study suggested that luminance requirements for conspicuity are related to the visual complexity of the scene, it provided neither practical methods of measuring complexity nor definitive levels of required luminance. It appears that visual complexity is multidimensional and that from a practical perspective it may be adequate to identify low complexity locations where degraded signs are adequate and high complexity locations which pose special problems.

\section{Purpose}

The research reported in the current study had 2 distinct goals. One goal was to advance the earlier work of cole and Jenkins (1980) and Mace et al. (1982) by developing a practical procedure for categorizing visual complexity of highway scenes. The other goal was to determine reasonable luminance standards for yellow warning signs for different levels of background complexity (given a procedure for categorizing scenes with respect to visual complexity).

The study restricted itself to categorization of complexity in nighttime highway scenes. It is believed that the principles of nighttime visual complexity also apply to daytime complexity, and any scales developed for rating complexity would be applicable to daylight and darkness. However, the complexity rating of a scene at night might be different from the scene's daytime categorization. Because of Iimitations on time and resources this study was restricted to conspicuity of the yellow diamond warning sign in night scenes. Only levels of Type II sheeting (engineering grade and degraded engineering grade) could be tested. Given these Iimitations and the positive nature of the results, it is hoped that further work will be done testing daytime and nighttime conspicuity of different sign materials and colors.

\section{MEASUREMENT OF VISUAL COMPLEXITY}

\section{A. Approaches to the Measurement of Complexity}

A number of approaches exist for the measurement of visual complexity. Since the interest in complexity is as a valid predictor of sign recognition, one approach might be to use a criterion measure, e.g., sign recognition distance. For example, sites where drivers don't recognize signs until they are within 500 ft $(150 \mathrm{~m})$ are, by definition, of high complexity. While such a measure would have the greatest accuracy, it would not be readily usable. First, a standard sign would have to be placed at each location to ensure that performance was not affected by the condition of the existing sign. Second, a group of unalerted subjects would be needed to obtain the recognition distances. Maintenance personnel could not do this because they would know the location of the sign. A measure obtained using knowledgeable subjects would reflect threshold perception (i.e., where a sign can be recognized) rather than conspicuity (i.e., where a sign will be recognized).

From a practical perspective it is clearly more desirable to have a procedure for measuring complexity which requires neither a sign nor a naive observer. The approach used by Jenkins (Note 1) was to rank order scenes according to clutter. Although a rank order measurement is adequate to demonstrate the effect of visual complexity, it cannot be used in practice because a scene's complexity rank is highly dependent on the sample of sites ranked. If the sample were changed, a site could change from high to low complexity or vice versa.

The measurement approach in the work of Mace et al. (1982) was to grade scenes on a large number of $v$ isual and photometric variables and use a regression formula to predict driver performance. Visual variables included counts of point sources of light; number of medium and large bright, extended light sources; and uniformity and detail of visual areas. Distracting things, traffic signs, number of lanes, etc., were also counted. Photometric variables included measurements of sign luminance, surround 1 uminance, and external and internal contrast. This work represented a significant step toward the measurement of real world complex scenes, but the procedures for scoring many of these variables were cumbersome. ClearIy, a smaller, simpler set of variables was needed if measurement of complexity were to have practical application. 


\section{B. Development of Complexity Scales}

One approach to the development of complexity scales would be to base them analytically on the principles from the literature of target detection discussed earlier. Another approach would be to factor-analyze the large domain of visual and photometric variables which have been shown to correlate with sign recognition (Mace et al., 1982). Both approaches were used to develop the complexity scales evaluated in this research. The factor analysis procedure and results are given in appendix $A$.

The 5 factors suggested by that analysis were:

\section{Ambiguity of Road Orientation}

If the road surface ahead is visible or roadside areas have detail, the direction of the road is likely to be clearer than if the road and roadside are not illuminated. Street lights and other luminaires provide a cue to the path of the road and a source of light to make the road surface and roadside areas visible. Parked cars also can reduce ambiguity about where the road goes.

\section{Presence of Distracting Visual} objects in scene 2

Drivers are less likely to see signs when they are distracted. A scene is distracting if it has medium or large bright lights and reflections to the left of the road or to the right of the cone. In the most distracting scenes, the cone may also have bright lights, bright reflections, and objects made visible by the lights. If something is dominant or unusual, it will add to the distraction. Although traffic signs along the road may themselves attract attention, they should not be considered distracting. Traffic signs are considered next. Headlights of oncoming cars also should not be considered here.

\section{Presence of other Traffic Signs}

Only the cone should be considered. Any traffic sign or object that looks like a traffic sign should be counted except signs immediately identified as no-parking signs. If there is uncertainty about whether something is a traf- fic sign, the sign should be counted. If more than 2 signs appear on 1 pole, the group should be counted as only 2 signs.

\section{Uniformity and Brightness of Cone ${ }^{3}$}

The cone becomes bright as it fills with bright lights and bright reflections. Signs are easier to recognize in a dark, uncluttered cone because contrast is increased and competition from the lights, reflections, and other detail is reduced.

\section{Heavy Demand from Driving Task}

Drivers' ability to detect and recognize traffic signs deteriorates as the demands of driving increase. The demands increase with the number of lanes and the number of vehicles traveling in the same direction. Many pinpoint lights to the left and right of the cone also increase the demands. Pedestrians and intersections controlled by traffic signals or signs add to the difficulty.

In addition to these 5 factors, 4 dimensions of visual complexity were defined based upon principles of detection reviewed by Mace and Pollack (1983) and referenced in chapter II. Two basic principles to which these dimensions related are: the brighter the visual noise, the lower the contrast and the poorer the visual performance; and the closer the visual noise to the target, the poorer the visual performance. The 4 dimensions were:

\section{Total Amount of Visual Detail in Scene}

Detail is anything that can be seen against the darkness. It includes lights and objects that the lights illuminate. The entire field of view should be considered, including the road, the horizon, and the area on both sides of the road.

7. Brightness of Visual Detail in Scene

Streetlights, internally 1 ighted signs, bright billboards, car lights, parking lot lights, lighted store windows, and bright reflections from glass and metal are examples of bright visual detail.

2 The scene is everything a driver sees when looking at an area through the windshield.

3 The cone is the portion of the right side of the road where a driver normally looks for roadside traffic signs. 


\section{Amount of Visual Detail in Cone}

Only lights and objects visible in the cone should be considered. Much detail may be visible in the scene, but very little in the cone. For example, a bridge approach may be lighted to the degree where one can see pavement seams. The horizon may be cluttered with lights. The cone, however, can include just a barely discernible bridge railing. Conversely, a dark country road with a lighted service station in the cone may exhibit little scene detail, but above average cone detail.

\section{Brightness of Visual Detail in Cone}

The amount of visual detail in the cone should be considered; then an estimate should be made about how much of it is bright. The cone becomes bright as it fills with bright lights and bright reflections. Signs are easier to recognize in a dark, uncluttered cone because contrast is increased and competition from the lights, reflections, and other detail is reduced.

In addition to the 5 factors and 4 dimensions, several attempts were made to obtain a single visual complexity scale. One, scale 10, asked the rater to judge the ease or difficulty of seeing a traf$f i c$ sign in a scene; another, scale 11 , asked for an estimate of how many glances might be required to recognize a traffic sign. With the factors and dimensions, these made 11 scales from which the final complexity rating procedure would be established.

The factor scales and other scales are clearly not independent of each other despite the fact that an orthogonal factor analysis procedure was used.

Two orthogonal dimensions appear to be suggested by the factors and literature. One is related to time: how much time the driver has to look for signs and how much time he needs. The items on the factor Ambiguity of Road Orientation appear related to concepts of familiarization and perceptual organization. Parked vehicles, luminaires, and road geometry help organize scenes such that search time is reduced by 1 imiting the area which must be searched. The number of lanes, intersections, and other vehicles make the task of driving more difficult and limit the time available to search for signs.

A second orthogonal dimension underlying the domain of visual complexity relates to all items affecting visual noise and masking sign recognition.
Items on the factor Uniformity and Brightness of Cone are clearly parts of this dimension of complexity. The factors Distracting Objects and other Traffic Signs probably mediate complexity through both of these 2 fundamental dimensions by occupying the driver's available time and masking the presence of the target sign.

\section{Laboratory study - Original Scenes}

Following a pilot test which contributed to the refinement of the 11 scales for rating complexity and the instructions for their use, a laboratory study was conducted to compare their validity and reliability. Subjects for this study were 32 employees of New Jersey Department of Transportation (NJDOT) in Trenton, New Jersey. The scenes on which the scales were used were the same 80 scenes used in the earlier study (Mace et al., 1982).

Subjects were divided into 2 groups: one group of 13 subjects used the 5 factors (scales 1 to 5 ) and general scale 10 to evaluate the complexity of 60 scenes, while the other group of 19 subjects used the 4 dimensions (scales 6 to 9 ) and general scale 11 to rate visual complexity of the same 60 scenes. The remaining 20 of the 80 original scenes were used for training and practice.

Multiple correlations were computed using a forward stepwise procedure for the 5 factors, the 4 dimensions, and all 11 scales combined ( 2 general scales included). A multiple correlation using all 5 factors produced an $\mathrm{R}^{2}$ of .153 , while a multiple $R$ based upon just 2 factors -- scales 3 and 4 -- produced an $\mathrm{R}^{2}$ of .147 , suggesting that these 2 factors alone were adequate. Similarly, all 4 dimensions produced an $\mathrm{R}^{2}$ of .154 , while 3 dimensions -- scales 6,8 , and 9 -- produced an $\mathrm{R}^{2}$ of .151 , suggesting that scale 7 may not be necessary. Finally all 11 variables resulted in an $\mathrm{R}^{2}$ of .271; however, equations based upon only 4 or 5 variables produced $R^{2}$ 's of about . 23 .

It is difficult to eliminate any variable based upon the validity of multiple correlations without specifying which variables will be used in a predictive equation. Because of suppressor effects, a variable which may not contribute to the predictive validity of one group of variables may contribute substantially when used with another group. Examination of a large number of regression equations suggested that scales $1,6,7$, and the 2 general scales could probably be eliminated. Regression equations made 


\section{FIELD VALIDATION OF VISUAL COMPLEXITY}

up of 4 or 5 of the remaining 6 scales produced $\mathrm{R}^{2}$ 's of about .22 . However, since the available criterion was obtained in a laboratory task, the possibility existed that the validities of the scales obtained from a field test could be markedly different. Therefore, decisions were made to combine scale 4 and scale 9 into one scale measuring brightness in the cone, to drop the 2 general scales and try again with a new one, and to drop scale 1 . This would result in 8 scales for further testing in a field study of 24 sites.

It was desirable to drop scale 1 (Ambiguity of Road orientation) for reasons other than its apparent validity. First, because the number of sites to be included in the field study was relatively small, sites with varying levels of ambiguity could not be tested at different levels of other critical site variables, e.g., brightness, driving difficulty, and distractions. Second, and most important, sites with high ambiguity often have geometric abnormalities which would affect available luminance. Signs of equal specific luminance change their level of brightness when placed at different geometric relationships to the driver. In the field study, it was desirable to approach each sign location along a straight and level road to minimize the effect geometry can have on available luminance.

There are pros and cons to the use of so many scales. The greatest single objection is that unless a large number of sites are tested, chance alone may produce significant validities. On the other hand, the research was exploratory and it did not seem desirable to limit the study to the testing of a few scales which were not thoroughly investigated. Even though we hypothesized only two fundamental underlying dimensions to the domain of complexity, we believed it could take a number of scales to account for the variance in this domain.

The rating scales that were retained from the analyses of the laboratory data were combined into a single instrument to be used for further testing. This form is shown in figure 1. The scales were rewritten and simplified for field use. Responses could be made simply by circling an answer. Scale values were intentionally omitted to emphasize the content of responses. The accompanying training materials are provided in appendix $B$.

\section{A. General Experimental Procedure}

The study described in this chapter was designed to develop a valid and reliable procedure for rating visual complexity, to compare photographic versus field rating techniques, and to establish luminance standards for high and low complexity locations.

The study was conducted in 2 phases using a set of 24 sites located on a 24$\mathrm{mi}(38.4-\mathrm{km})$ test loop in and around Trenton, New Jersey. In Phase I, subjects were asked to rate the sites on the 8 complexity scales. One group of 12 rated the sites in the field, and another group of 15 rated the sites pictured in projected transparencies. During Phase II, yellow diamond warning signs were installed at the test sites, and 15 subjects drove the loop while their recognition and legibility distances to each sign were recorded.

\section{B. Site selection}

A number of considerations influenced site selection. In addition to the primary objective of selecting sites distributed across the range of low to high complexity, other factors had to be considered such as the limitations of road geometry on sight distance, hazardous traffic conditions for observation vehicles, and the time required for subjects to traverse all of the sites.

Initially 30 sites were selected along a $28-\mathrm{mi}(44.8-\mathrm{km})$ loop beginning and ending at the Traffic Research Building of the NJDOT in Trenton. The sites were then evalauted against the following criteria:

- There had to be at least $1500 \mathrm{ft}$ (450 m) of unobstructed sight distance to the potential sign location for Phase II.

- The potential sign location could not be less than $300 \mathrm{ft}(90 \mathrm{~m})$ beyond a signalized intersection because drivers stopped at a red light would have an advantage from increased observation time.

- Light sources (signs, store fronts, street lights, etc.) contributing to scene brightness and complexity had to remain relatively constant from dusk until at least 11:00 p.m. to allow multiple subject runs per evening. 
1. How distracting is the scene?

$\begin{array}{ccc}\text { very } & \text { not very } & \text { not } \\ \text { distracting distracting } & \text { average } & \text { distracting }\end{array}$

2. How many traffic signs are in the cone?

five

or more

four

three

two

one or none

3. How much detail is visible in the scene?

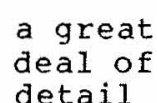

detail average little
detail

very little detail

4. How many bright sources are in the scene?

$$
\text { very }
$$$$
\text { many }
$$

many

average

few

very

few

5. How much detail is visible in the cone?

$\begin{array}{lcccc}\text { a great } & \text { a lot } & \text { little } & \text { very } \\ \text { deal of } & \text { of } & \text { average } & \text { detail } & \text { little } \\ \text { detail } & \text { detail } & & & \text { detail }\end{array}$

6. How bright is the cone?

\begin{tabular}{|c|c|}
\hline $\begin{array}{l}\text { very } \\
\text { oright }\end{array}$ & bright \\
\hline
\end{tabular}

7. How demanding would driving be at this location?

very
demanding demanding average demanding easy

8. To be easily spotted, how bright would a new traffic sign have to be?

$\begin{array}{llcl}\text { very } & \text { not } & \text { dim } \\ \text { bright } & \text { bright } & \text { average } & \text { bright }\end{array}$

Figure 1. Complexity evaluation form. 
After review, the number of sites was reduced to 24 , and the driving loop was shortened to $24.5 \mathrm{mi}(39.2 \mathrm{~km})$. These sites were photographed (without signs), and all 24 transparencies were used in Phase I. Prior to Phase II, sites 8 and 23 were dropped because of difficulty in finding a suitable location for the experimental sign. Phase II data was collected on the remaining 22 sites, but 3 of them were eliminated from the final analysis because foliage affected sign visibility. Table 1 provides a summary of site characteristics and denotes those sites that were dropped for various reasons.

\section{Phase I: The Measurement of Complexity}

The purpose of this phase of the study was to compare a procedure whereby the visual complexity of sites is measured in the field and one whereby complexity is measured from projected transparancies. The primary basis of evaluation would be scale reliability with $v a l i d i t y$ to be considered only if both procedures gave equally reliable results.

\section{Site Preparation}

During the test site selection procedure, each site was surveyed to establish a potential sign location and provide a constant vantage point for rating complexity. The potential sign location was referenced with a small, high intensity reflector installed about $4 \mathrm{ft}(1.2 \mathrm{~m})$ above ground level and within $3 \mathrm{ft}(.9 \mathrm{~m})$ of the curb. The site evalution distance, $500 \mathrm{ft}(150 \mathrm{~m})$ prior to the potential sign location, was designated with a small patch of reflective pavement tape placed on the shoulder or curb. 4 Each site was photographed at night (from the evaluation distance) with a tripodmounted $35 \mathrm{~mm}$ camera positioned in the right lane at approximate driver eye height and position. The camera was aimed straight down the lane. No supplemental illumination was used.

\section{Test Vehicles}

The road evaluation segment of Phase I required a large capacity vehicle equipped with flashing yellow overhead warning lights directed to the rear. An NJDOT commuter van was used on 2 successive evenings to transport subjects through the sites. Each session included 7 to 8 subjects, 2 experimenters, and a dri- ver. Subjects were supplied with rating forms, clipboards, pencils, and a small flashlight to illuminate the response forms.

\section{Subjects}

Phase I subjects were selected from a pool of NJDOT professional employees. Twelve subjects with an age range of 22 to 55 were assigned to the photographic evaluation group, and 15 subjects ranging in age from 23 to 62 constituted the road evaluation group. All subjects were residents of the Trenton area and consequently had some familiarity with the routes and locations of the test sites.

\section{Data Collection Procedure}

The photo evaluation portion of Phase I was conducted in a conference room of NJDOT Traffic Research Building. Subjects were assembled in a classroom setting facing a projection screen, and were supplied with instruction sheets, evaluation forms, pencils, a clipboard, and a small flashlight. Following a brief introduction to the study, subjects were given time to read the instructions (appendix B) and were solicited for questions. They were then shown 7 practice slides which illustrated aspects of complexity described in the instructions, e.g. , cone brightness, scene brightness, etc. Finally, transparencies of the 24 test sites were shown in sequence, allowing approximately 2 mintues per slide. Subjects recorded their ratings of each over the 8 evaluation scales on their response sheets.

The field evaluation group received the same instructions and practice slides in the same setting as the photo evaluation group. Instead of seeing slides of the 24 sites, however, they boarded a 12passenger van and were driven to each site to evaluate it. As the driver approached each site, an experimenter in the front of the vehicle called his attention to a marked spot on the pavement denoting the 500-ft $(150-\mathrm{m})$ evaluation distance. The driver would stop at the mark in the rightmost lane and turn on yellow, warning lights as a safety precaution. The vehicle headlights were on low beam, and the flashing yellow Iights were adjusted to prevent their light from illuminating the scene in front of the vehicle. The van remained in the lane for $30 \mathrm{sec}$ while the subjects observed the site; then the driver

\footnotetext{
4 A site evaluation distance of $500 \mathrm{ft}(150 \mathrm{~m})$ was selected as the most appropriate based upon judgments of project personnel. Evaluation distances of 300 to $600 \mathrm{ft}$ $(90$ to $180 \mathrm{~m})$ in $50-\mathrm{ft}(15-\mathrm{m})$ increments were tested in a small preparatory study and the 500-ft $(150-\mathrm{m})$ distance was chosen through consensus.
} 
After review, the number of sites was reduced to 24 , and the driving loop was shortened to $24.5 \mathrm{mi}(39.2 \mathrm{~km})$. These sites were photographed (without signs), and all 24 transparencies were used in Phase I. Prior to phase II, sites 8 and 23 were dropped because of difficulty in finding a suitable location for the experimental sign. Phase II data was collected on the remaining 22 sites, but 3 of them were eliminated from the final analysis because foliage affected sign visibility. Table 1 provides a summary of site characteristics and denotes those sites that were dropped for various reasons.

\section{Phase I: The Measurement of Complexity}

The purpose of this phase of the study was to compare a procedure whereby the visual complexity of sites is measured in the field and one whereby complexity is measured from projected transparancies. The primary basis of evaluation would be scale reliability with validity to be considered only if both procedures gave equally reliable results.

\section{Site Preparation}

During the test site selection procedure, each site was surveyed to establish a potential sign location and provide a constant vantage point for rating complexity. The potential sign location was referenced with a small, high intensity reflector installed about $4 \mathrm{ft}(1.2 \mathrm{~m})$ above ground level and within $3 \mathrm{ft}(.9 \mathrm{~m})$ of the curb. The site evalution distance, $500 \mathrm{ft}(150 \mathrm{~m})$ prior to the potential sign location, was designated with a small patch of reflective pavement tape placed on the shoulder or curb. ${ }^{4}$ Each site was photographed at night (from the evaluation distance) with a tripodmounted $35 \mathrm{~mm}$ camera positioned in the right lane at approximate driver eye height and position. The camera was aimed straight down the lane. No supplemental illumination was used.

Test Vehicles

The road evaluation segment of Phase I required a large capacity vehicle equipped with flashing yellow overhead warning lights directed to the rear. An NJDOT commuter van was used on 2 successive evenings to transport subjects through the sites. Each session included 7 to 8 subjects, 2 experimenters, and a dri- ver. Subjects were supplied with rating forms, clipboards, pencils, and a small flashlight to illuminate the response forms.

\section{Subjects}

Phase I subjects were selected from a pool of NJDOT professional employees. Twelve subjects with an age range of 22 to 55 were assigned to the photographic evaluation group, and 15 subjects ranging in age from 23 to 62 constituted the road evaluation group. All subjects were residents of the Trenton area and consequently had some familiarity with the routes and locations of the test sites.

\section{Data Collection Procedure}

The photo evaluation portion of Phase I was conducted in a conference room of NJDOT Traffic Research Building. Subjects were assembled in a classroom setting facing a projection screen, and were supplied with instruction sheets, evaluation forms, pencils, a clipboard, and a small flashlight. Following a brief introduction to the study, subjects were given time to read the instructions (appendix B) and were solicited for questions. They were then shown 7 practice slides which illustrated aspects of complexity described in the instructions, e.g., cone brightness, scene brightness, etc. Finally, transparencies of the 24 test sites were shown in sequence, allowing approximately 2 mintues per slide. Subjects recorded their ratings of each over the 8 evaluation scales on their response sheets.

The field evaluation group received the same instructions and practice slides in the same setting as the photo evaluation group. Instead of seeing slides of the 24 sites, however, they boarded a 12passenger van and were driven to each site to evaluate it. As the driver approached each site, an experimenter in the front of the vehicle called his attention to a marked spot on the pavement denoting the 500-ft $(150-\mathrm{m})$ evaluation distance. The driver would stop at the mark in the rightmost 1 ane and turn on yellow, warning lights as a safety precaution. The vehicle headlights were on low beam, and the flashing yellow lights were adjusted to prevent their light from illuminating the scene in front of the vehicle. The van remained in the lane for $30 \mathrm{sec}$ while the subjects observed the site; then the driver

4 A site evaluation distance of $500 \mathrm{ft}(150 \mathrm{~m})$ was selected as the most appropriate based upon judgments of project personnel. Evaluation distances of 300 to $600 \mathrm{ft}$ $(90$ to $180 \mathrm{~m})$ in $50-\mathrm{ft}(15-\mathrm{m})$ increments were tested in a small preparatory study and the $500-\mathrm{ft}(150-\mathrm{m})$ distance was chosen through consensus. 
Table 1. Site characteristics.

\begin{tabular}{|c|c|c|c|c|c|c|}
\hline Site & $\begin{array}{c}\text { Highway } \\
\text { Classification } \\
\end{array}$ & $\begin{array}{l}\text { Speed } \\
\text { Limit } \\
\text { (mph) }\end{array}$ & $\begin{array}{c}\text { Area } \\
\text { Classification } \\
\end{array}$ & $\begin{array}{c}\text { Traffic } \\
\text { Volume } \\
\text { (late evening) } \\
\end{array}$ & Sign Location & Characterisitics \\
\hline 1 & $\begin{array}{l}\text { Four-lane } \\
\text { minor arterial }\end{array}$ & 40 & Light commercial & Low & $\begin{array}{l}\text { Adjacent to a large } \\
\text { office building and } \\
\text { across from a small } \\
\text { shopping center }\end{array}$ & \\
\hline 2 & $\begin{array}{l}\text { Four-lane } \\
\text { minor arterial }\end{array}$ & 30 & Light commercial & Low & $\begin{array}{l}\text { Adjacent to a restau- } \\
\text { rant and across from a } \\
\text { shopping center }\end{array}$ & \\
\hline 3 & $\begin{array}{l}\text { Four-lane } \\
\text { minor arterial }\end{array}$ & 40 & Residential & Low & Street corner & $\begin{array}{l}\text { Sign approximately } \\
1 / 8 \text { th obscured on } \\
\text { right by foliage }\end{array}$ \\
\hline 4 & $\begin{array}{l}\text { Four-lane } \\
\text { parkway (freeway) }\end{array}$ & 50 & $\begin{array}{l}\text { Park (no pedes- } \\
\text { trian access to } \\
\text { freeway) }\end{array}$ & Medium & $\begin{array}{l}\text { Within fenced right of } \\
\text { way, just beyond a } \\
\text { pedestrian overpass }\end{array}$ & $\begin{array}{l}\text { Sign approximately } \\
1 / 4 \text { th obscured on } \\
\text { top corner by foli- } \\
\text { age. Site not used } \\
\text { in Phase II analysis }\end{array}$ \\
\hline 5 & $\begin{array}{l}\text { Two-lane } \\
\text { major arterial } \\
\text { with no shoulders }\end{array}$ & 25 & Medium commercial & High & $\begin{array}{l}\text { Adjacent to a garage } \\
\text { and across from a super- } \\
\text { market }\end{array}$ & $\begin{array}{l}\text { Sign momentarily } \\
\text { illuminated at ap- } \\
\text { proximately } 1200 \\
\text { ft ( } 360 \mathrm{~m}) \text { as low } \\
\text { beams were elevated } \\
\text { by a RR grade }\end{array}$ \\
\hline
\end{tabular}

\begin{tabular}{|c|c|c|c|c|c|c|}
\hline 6 & $\begin{array}{l}\text { Two- } 1 \text { ane } \\
\text { major arterial }\end{array}$ & 25 & $\begin{array}{l}\text { Light commercial } \\
\text { and residential }\end{array}$ & High & Adjacent to a house & \\
\hline 7 & $\begin{array}{l}\text { Four-lane } \\
\text { minor arterial }\end{array}$ & 35 & Light commercial & Medi um & Right of parking lane & $\begin{array}{l}\text { Sign } 1 / 4 \text { th obscured } \\
\text { by foliage. Site } \\
\text { not used in Phase II } \\
\text { analysis }\end{array}$ \\
\hline 8 & $\begin{array}{l}\text { Four-lane } \\
\text { minor arterial }\end{array}$ & 35 & Light commercial & Medium & Not used & $\begin{array}{l}\text { Site eliminated for } \\
\text { Phase II }\end{array}$ \\
\hline 9 & $\begin{array}{l}\text { Four-lane } \\
\text { minor arterial }\end{array}$ & 35 & Light commercial & Medium & $\begin{array}{l}\text { Adjacent to a small } \\
\text { shopping mall entrance }\end{array}$ & \\
\hline 10 & $\begin{array}{l}\text { Two- } 1 \text { ane } \\
\text { minor arterial }\end{array}$ & 35 & Residential & Medium & $\begin{array}{l}\text { Sign located within a } \\
\text { cluster of other signs }\end{array}$ & $\begin{array}{l}\text { Sign } 1 / 8 \text { th obscured } \\
\text { on right by foliage }\end{array}$ \\
\hline
\end{tabular}


Table 1. Site characteristics (continued).

\begin{tabular}{|c|c|c|c|c|c|c|}
\hline Site & $\begin{array}{c}\text { Highway } \\
\text { Classification }\end{array}$ & $\begin{array}{l}\text { Speed } \\
\text { Limit } \\
\text { (mph) } \\
\end{array}$ & $\begin{array}{c}\text { Area } \\
\text { Classification } \\
\end{array}$ & $\begin{array}{c}\text { Traffic } \\
\text { Volume } \\
\text { (late evening) } \\
\end{array}$ & Sign Location & $\begin{array}{l}\text { Special } \\
\text { Characterisitics }\end{array}$ \\
\hline 11 & $\begin{array}{l}\text { Four-lane minor } \\
\text { arterial }\end{array}$ & 45 & Rural & Low & $\begin{array}{l}\text { Next to exit lane from a } \\
\text { restaurant. Somewhat } \\
\text { greater of }{ }^{2} \text { set than } \\
\text { normal }\end{array}$ & $\begin{array}{l}\text { Slight dogleg in } \\
\text { road reduced recog- } \\
\text { nition distance. } \\
\text { Site not used in } \\
\text { Phase II analysis }\end{array}$ \\
\hline 12 & $\begin{array}{l}\text { Four-lane minor } \\
\text { arterial }\end{array}$ & 45 & Rural & Medium & $\begin{array}{l}\text { Adjacent to a restaurant } \\
\text { and upstream of a major } \\
\text { intersection }\end{array}$ & \\
\hline 13 & $\begin{array}{l}\text { Two-lane major } \\
\text { arterial with } \\
\text { turning lane }\end{array}$ & 40 & Heavy commercial & $\mathrm{High}$ & $\begin{array}{l}\text { Between a gas station } \\
\text { and a restaurant }\end{array}$ & \\
\hline 14 & $\begin{array}{l}\text { Two-lane major } \\
\text { arterial with } \\
\text { turning lane }\end{array}$ & 40 & Heavy commercial & $\mathrm{High}$ & Adjacent to a restaurant & \\
\hline 15 & $\begin{array}{l}\text { Two-lane major } \\
\text { arterial with } \\
\text { turning lane }\end{array}$ & 40 & Medium commercial & High & $\begin{array}{l}\text { Adjacent to a car } \\
\text { dealership }\end{array}$ & $\begin{array}{l}\text { Sign appeared } \\
\text { siihouetted against } \\
\text { the sky }\end{array}$ \\
\hline 16 & $\begin{array}{l}\text { Two-lane major } \\
\text { arteriai with } \\
\text { turning lane }\end{array}$ & 40 & Medium commercial & $\mathrm{High}$ & $\begin{array}{l}\text { Adjacent to a car } \\
\text { dealership }\end{array}$ & $\begin{array}{l}\text { Legibility of the } \\
\text { sign may have been } \\
\text { influenced by glare }\end{array}$ \\
\hline 17 & $\begin{array}{l}\text { Two-lane major } \\
\text { arterial with } \\
\text { turning lane }\end{array}$ & 40 & Heavy commercial & High & $\begin{array}{l}\text { Adjacent to a shopping } \\
\text { center entrance and near } \\
\text { a major intersection }\end{array}$ & \\
\hline 18 & $\begin{array}{l}\text { Four-lane major } \\
\text { arterial }\end{array}$ & 40 & $\begin{array}{l}\text { Light commercial/ } \\
\text { rural }\end{array}$ & Low & $\begin{array}{l}\text { Adjacent to an office } \\
\text { building }\end{array}$ & \\
\hline 19 & $\begin{array}{l}\text { Four-lane major } \\
\text { arterial }\end{array}$ & 55 & Medium commercial & Low & $\begin{array}{l}\text { Adjacent to car } \\
\text { dealership }\end{array}$ & \\
\hline 20 & $\begin{array}{l}\text { Four-lane major } \\
\text { arterial }\end{array}$ & 45 & Light commercial & Low & $\begin{array}{l}\text { Just beyond a jug-handle } \\
\text { intersection }\end{array}$ & \\
\hline 21 & $\begin{array}{l}\text { Four-lane major } \\
\text { arterial }\end{array}$ & 40 & Medium commercial & Low & $\begin{array}{l}\text { Adjacent to a used car } \\
\text { lot }\end{array}$ & \\
\hline 22 & $\begin{array}{l}\text { Four-lane minor } \\
\text { arterial }\end{array}$ & 40 & Medium commercial & Medi um & $\begin{array}{l}\text { Adjacent to a car } \\
\text { dealership }\end{array}$ & \\
\hline 23 & $\begin{array}{l}\text { Four-lane minor } \\
\text { arterial }\end{array}$ & 40 & Medium commercial & Medium & Not used & $\begin{array}{l}\text { Site eliminated from } \\
\text { Phase II }\end{array}$ \\
\hline 24 & $\begin{array}{l}\text { Four-lane minor } \\
\text { arterial }\end{array}$ & 40 & Medium commercial & Medi um & $\begin{array}{l}\text { Entrance to a shopping } \\
\text { center }\end{array}$ & \\
\hline
\end{tabular}

Note: $1 \mathrm{mi} / \mathrm{h}=1.6 \mathrm{~km} / \mathrm{h}$. 
would pull to the shoulder to clear traffic and remain until all subjects had completed their evaluation forms for the site. The field evaluation was conducted over 2 evenings between the hours of 9:00 and 11:00 p.m.

D. Phase II: The Acquisition of Sign Recognition and Legibility Distances

The purpose of this phase of the study was twofold: first, to provide a criterion (recognition distance) which could be used as a surrogate measure of visual complexity in the development of a composite complexity scale; second, to establish differential luminance requirements for the yellow diamond warning sign at low and high complexity locations.

\section{Site Preparation}

In preparation for Phase II, the small reflectors designating potential sign location were removed, and replaced with 30 -in $(75-\mathrm{cm})$ yellow diamond warning signs mounted at a height of $7 \mathrm{ft}(2.1 \mathrm{~m})$ (measured from ground to the bottom corner of the sign). The signs were mounted on $11 / 8$-in $(2.8-\mathrm{cm})$ square galvanized steel posts and bolted with wingnuts for rapid removal. Sign posts were positioned within $3 \mathrm{ft}(.9 \mathrm{~m})$ of the curb and/or in line with existing sign posts, with offsets from the travel lane as noted in table 1 .

\section{Variables}

In addition to site complexity, the independent variable of specific sign luminance was included so that luminance requirements could be determined. Three levels of sign brightness were employed. Signs constructed with new Type II sheeting (engineering grade) provided the highest luminance condition. Artificial degradation with a silk-screened dot pattern was used to produce signs degraded to approximately 72 percent and 36 percent of the Federally specified level of new Type II signs. Degradation levels were verified with a retroreflectometer.

Dependent variables included sign recognition distance and sign legibility distance. Recognition distance is the distance from a sign to where it can be identified as a yellow diamond sign. Legibility distance is the distance from the sign to where its entire legend can be identified. To control for the effects of experience and learning, sign legends had to be novel and varied within experimental sessions. Various sign types and messages were considered from the standpoints of suitability for the study objectives and effect upon the indiginous traffic. The selected signs were $30-$ in $(75-\mathrm{cm})$, standard yellow, diamond warning signs, each bearing one of 6 single word legends. The legends were TRUCK, CROSS, DRIVE, SCHOOL, OUTLET, and STREET. Letters were 5 in $(12.5 \mathrm{~cm})$ high, series D (U.S. Department of Transportation, 1972).

Test Vehicles

Three vehicles, each equipped with a calibrated digital measuring instrument (DMI) and a battery powered tape recorder, were used on each of 3 evenings. The vehicles included a $1980 \mathrm{mid}-\mathrm{size}$ sedan, a 1976 full-size van, and a 1982 mid-size station wagon. All vehicles were equiped with halogen headlamps aimed to State inspection specifications. The headlamps were used at low beam. Passengers in each vehicle for each subject run included the subject/driver and an experimenter who recorded subject response distances. The DMI provided a digital readout of distance traveled in feet and was equipped with a button to freeze a display while the count progressed. Each time the subject indicated a sign recognition or sign legibility, the experimenter would freeze the display and record the last 4 digits on the tape recorder. An entry of the display was also made when the vehicle passed the sign location.

\section{subjects}

of 15 Phase II subjects, 3 were NJDOT employees. All were licensed drivers and residents of the Trenton area. The group ranged in age from 22 to 64 with a mean age of 38, and included 11 males and 4 females. Subjects were tested for visual acuity and color perception, and all had corrected acuity of $20 / 40$ or better with no color deficiencies.

\section{Experimental Design}

The Phase II design involved 2 independent variables, site complexity and sign brightness. Since the order of sites was fixed, the 3 levels of sign brightness were rotated 3 times over the course of the study. Each test site

5 The specified value for new Type II yellow sheeting is 50 SIA (U.S. Department of Transportation, 1979, section 633). The actual measured value was 65 SIA and the degraded forms averaged 36 SIA and 18 SIA. (SIA expressed as candelas per footcandle per square foot at . 2 degree observation angle and -4 degree entrance angle.) 
was observed by 5 independent subjects under each level of sign brightness, producing a total of 15 observations per site. Sign legends were systematically assigned to sites and brightness levels, and were completely rotated 3 times to avoid brightness-legend associations.

\section{Data Collection Procedure}

During Phase II, study subjects were processed at the rate of 5 per evening over 3 sequential evenings. Each subject drove an instrumented vehicle, and since only 3 vehicles were available, subjects were processed in 2 shifts each evening, with 3 departing from the NJDOT Traffic Research Building at 9:00 p.m. and 2 at 10:00 p.m. after the first run was completed.

Subjects met at the NJDOT building approximately one half hour before each run began and were tested for visual acuity and color perception. They were given a brief introduction to the project and were read the instructions included in appendix $C$. After familiarization with the vehicles and a question and answer session, they began the $24-\mathrm{mi}$ (38.4-km) loop.

Each vehicle contained a subject driver and an experimenter. The subject would call out "detect" when a yellow diamond warning sign was first spotted, and would read the legend as soon as possible. (In the case of signs containing a symbol, the subject would say "symbol.")

The experimenter was responsible for giving the subject directions regarding turns on the loop and for recording each detection and legibility response. When the subject would say "detect," the experimenter would push a button to freeze the digital readout on the DMI, and would read the last 4 digits into a hand held tape recorder. He would then restart the readout and repeat the process when the legend was read, and again when the vehicle crossed the point of sign location.

On responses to signs not part of the study, the latter 2 recordings were omitted. Upon reducing the data from recording to hard copy, the 4-digit response locations were subtracted from the sign location reading to give recognition and legibility data expressed in feet from the sign location.

\section{E. Results}

Analysis of Complexity Scales

Before analyzing the validity of the complexity scales with respect to their association with sign recognition distance, it is important to assess the reliability of the scales for evaluation of both the field and photo rating groups. Scenes were paired on the performance criterion before random assignment to 2 groups of 11 scenes. SpearmanBrown split-half reliability coefficients were then computed for each of the 8 scales for both groups of raters. These coefficients are shown in table 2 .

Table 2. Spearman-Brown reliability coefficient for each scale and 2 groups of raters.

Scale Photo Group Field Group

$\begin{array}{lll}1 & .27^{\star} & .82 \\ 2 & .81 & .87 \\ 3 & .80 & .79 \\ 4 & .91 & .97 \\ 5 & .56 * & .80 \\ 6 & .32^{\star} & .73 \\ 7 & .85 & .83 \\ 8 & .81 & .89 \\ .05 & & \end{array}$

In general, the use of the scales in the field had greater reliability than the use of the scales with photographs. All of the scales had statistically significant reliabilities in the field group. Three scales in the photo group had reliabilities which were not significant. Scale 1, which measured distractions, and scales 5 and 6 , which measured detail and brightness in the cone, appear to lack reliability when used with photographs.

A split-half reliability coefficient was also calculated for the recognition performance (Phase II) criterion. For this reliability estimate the scenes were split into the same 2 groups as before, and the average recognition distance for each subject at 1 group of locations was correlated with his scores at the second group of locations. The obtained coefficient was .88 , which is highly significant.

For regression analysis the sample of 22 sites was reduced to 19 . Sites 4, 7, and 11 were eliminated because of problems caused by foliage during recognition performance (Phase II), which confounded the criterion data. To determine the validity of each scale, zero-order correlations were computed with the performance criterion of recognition distance for each group of raters. These correlations are shown in table 3. Since the ratings of a second photo group were 
obtained after summer foliage changed the complexity of some scenes, as it had during recognition performance, it was expected that the ratings of this group would have higher validity. ${ }^{6}$ with the exception of scale 1 (How distracting is the scene?), this was the case. However, the validities for both photo groups were, in general, lower than the validity of the same scales rated by the road group. With the exception of scale 4 (bright sources in the scene), the variance accounted for by the other 7 scales in the road group ranged from .12 for scale 6 to .23 for scale 1 .

Table 3. Zero-order correlations of 8 complexity scales with performance criterion of recognition distance.

\begin{tabular}{|c|c|c|c|c|}
\hline Scale & & $\begin{array}{l}\text { Road } \\
\text { Group }\end{array}$ & $\begin{array}{c}\text { Photo } 1 \\
\text { Group }\end{array}$ & $\begin{array}{c}\text { Photo } 2 \\
\text { Group }\end{array}$ \\
\hline 1 & - & .48 & -.28 & -.10 \\
\hline 2 & - & .38 & -.26 & -.41 \\
\hline 3 & - & .40 & -.32 & -.39 \\
\hline 4 & - & .21 & -.39 & -.35 \\
\hline 5 & - & .45 & -.36 & -.34 \\
\hline 6 & - & .35 & -.33 & -.31 \\
\hline 7 & - & .45 & -.12 & -.28 \\
\hline 8 & - & .38 & -.23 & -.32 \\
\hline
\end{tabular}

Using a forward stepwise procedure, multiple regression equations were computed for both the road group from Phase $I$ and photo 2 group. Al1 8 scales were considered in the road group regression equations, but only the 5 scales which had significant reliability coefficients were included in the photo 2 group regression. With all 8 scales the $R$ for the road group was .83 . With 5 scales the $R$ for photo 2 group was only .54 . The $R$ for the photo 2 group was as high as .49 using only 2 scales -- 2 and 3 -which indicates there is little gained by using more than 2 photo rating scales. In terms of predicted variance $\left(R^{2}\right)$ the photo 2 group could account for a maximum 30 percent while the road group accounted for 69 percent. These results are summarized in table 4. These data suggest that a satisfactory formula is not avai1able using ratings from photographs. A formula with $4\left(R^{2}=.62\right)$ scales using ratings obtained on the road would appear most promising. The increase in validity with more than 4 variables would not seem to be worth the additional effort.
Table 4. $\mathrm{R}^{2}$ using varying numbers of scales from photo 2 and road groups.

\begin{tabular}{lcc}
$\#$ & $\begin{array}{c}\text { Photo } 2 \\
\text { Group }\end{array}$ & $\begin{array}{l}\text { Road } \\
\text { Group }\end{array}$ \\
\cline { 2 - 3 } 2 & .24 & .49 \\
3 & .27 & .56 \\
4 & .29 & .62 \\
5 & .30 & .65 \\
6 & --- & .69 \\
7 & --- & .69 \\
8 & --- & .69
\end{tabular}

The equations with the highest validities using 4 variables are shown below. Neither of these equations uses scale 6 , which had the lowest reliability. It is surprising to note that scale 4 , which had the lowest correlation with the recognition distance criterion, is represented in both equations. The fact is that an analysis of a large number of regression equations revealed that scale 4 is a critical predictor. Alternative equations using an equal number of predictors without scale 4 had dramatically lower validities.

The general model for 1 inear regression is $Y=a+b X$. Expanding this equation and substituting the actual weights for each scale in 2,4 variable equations result in the following formulas: ${ }^{7}$

Equation 1 :

$Y=2030+409 x_{3}+341 x_{4}+$

$\left(-543 x_{5}\right)+\left(-615 x_{7}\right)$.

Equation 2:

$\mathrm{Y}=1454+\left(-271 \mathrm{x}_{1}\right)+\left(-52 \mathrm{x}_{2}\right)+$ $297 x_{4}+\left(-162 x_{5}\right)$.

Examination of the weights for scales 3 and 4 indicates that both of these variables have positive weights while the other scales have negative weights.

Scales 3 and 4 , which measure detail and brightness of the scene, are suppressor variables. A suppressor variable improves the overall validity of a multiple regression equation, not from common variance with the criterion, but from variance it shares with the other predictors. The reader is referred to McNemar (1963) for a more thorough description of supressor variables.

\footnotetext{
6 Photo 2 group comprised of members of the research staff.

$7 x_{i}$ is the observed value for the $i$ th scale.
} 
Analysis of Recognition/Legibility Data

Tables 5 and 6 present a series of decriptive statistics representing subject recognition and legibility performance. Table 5 presents the mean recognition distance for each level of sign brightness and the 100 th percentile distance for medium and high brightness signs and high brightness signs only. Table 6 presents the mean legibility distance by each sign brightness level and all brightnesses combined.

In order to determine if the various sites produced statistically significant performance differences across the site complexity dimensions and to test the influence of sign brightness, a 2-way analysis of variance (AOV) was computed for recognition and legibility scores. Prior to calculating the AOV, the sites were categorized as high, medium, or low complexity. The categories were operationally defined based upon predicted recognition distances obtained from the 4-variable multiple regression equation (Equation 1). The 4 sites showing the longest predicted recognition distances were classified as low complexity, and the 4 sites with the shortest predicted recognition distances were classified as high complexity. Four medium complexity sites were randomly selected from the remaining pool. Random selection was used so that the middle group would represent all sites not judged high or low complexity. The predicted and actual means for each of these categories are shown in table 7 .

Table 7. Predicted versus actual recognition distances ( $f t$ ) (12 Sites).

\begin{tabular}{|c|c|c|}
\hline & Predicted & Actual \\
\hline $\begin{array}{l}\text { Low Complexity } \\
\text { (Mean for Sites } \\
6,9,18,19)\end{array}$ & 1158 & 1162 \\
\hline $\begin{array}{l}\text { Medium Complexity } \\
\text { (Mean for Sites } \\
5,12,14,17)\end{array}$ & 937 & 1030 \\
\hline $2,10,13,24)$ & 694 & 707 \\
\hline
\end{tabular}

Note: Predicted from the multiple $\mathrm{R}$ for scales $3,4,5$, and 7 .

Note: $1 \mathrm{ft}=.3 \mathrm{~m}$.

Table 8 shows the mean recognition distances for a 3 by 3 factorial design of visual complexity and sign brightness. As expected, an analysis of variance showed visual complexity was significant $(p<.0001)$ and sign brightness was significant $(p<.01)$. These cell means are plotted in figure 2. The interaction of sign brightness and visual complexity was not significant.

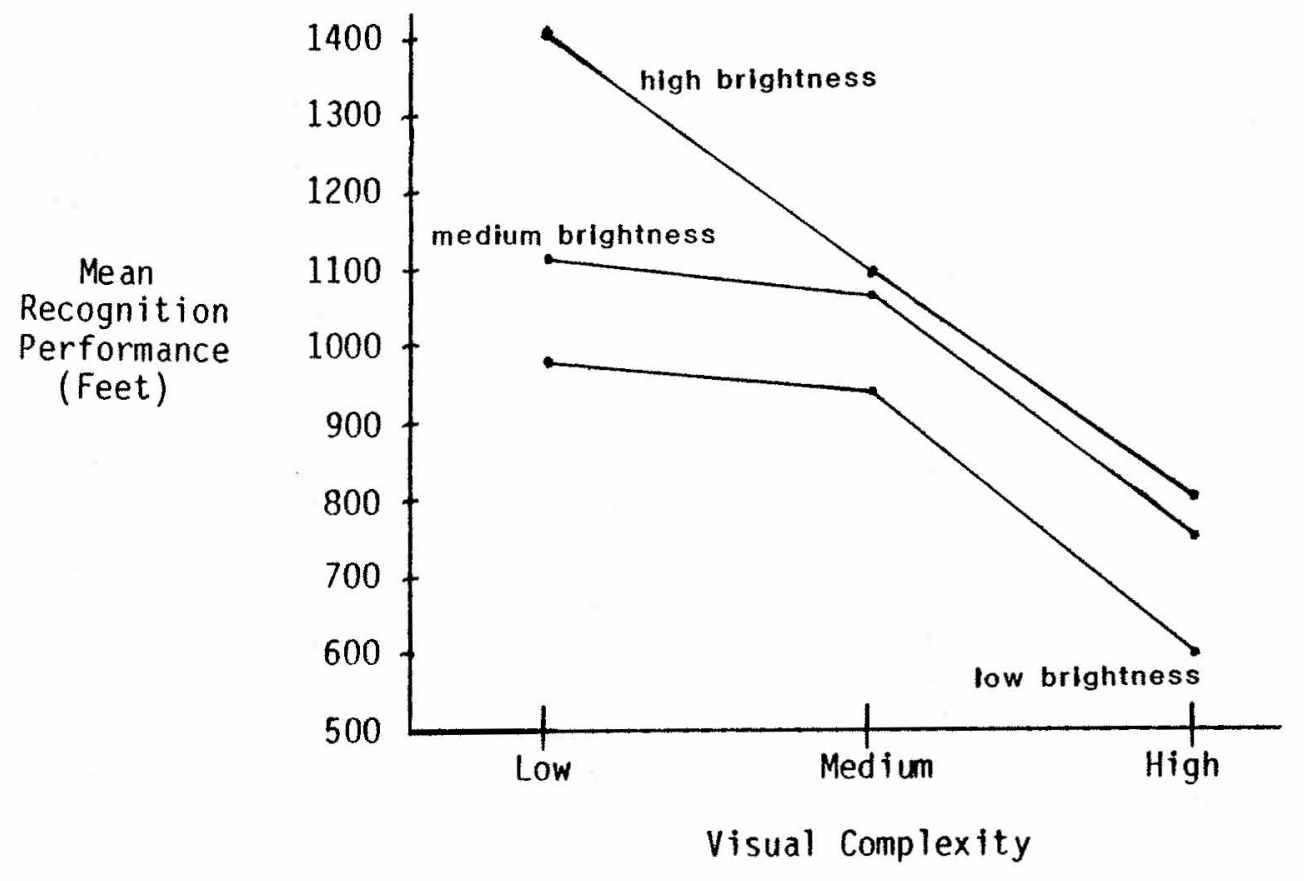

Figure 2. Recognition performance by visual complexity and sign brightness.

Note: $1 \mathrm{ft}=.3 \mathrm{~m}$. 
Table 5. Recognition distance ( $f t$ ) performance by site and sign brightness level.

\begin{tabular}{|c|c|c|c|c|c|c|c|}
\hline \multirow[b]{3}{*}{ Site } & & & & L umina & \multirow{2}{*}{\multicolumn{3}{|c|}{ 100th Percentile }} \\
\hline & \multicolumn{4}{|c|}{ Mean } & & & \\
\hline & Low & Medium & $\mathrm{High}$ & $\begin{array}{c}\text { All } \\
\text { Signs }\end{array}$ & $A 11$ & $\begin{array}{c}\text { Med. }{ }^{\prime} \\
\text { High }\end{array}$ & $\begin{array}{l}\text { High } \\
\text { only }\end{array}$ \\
\hline $\begin{array}{l}1 \\
2\end{array}$ & $\begin{array}{r}1062 \\
804\end{array}$ & $\begin{array}{l}779 \\
811\end{array}$ & $\begin{array}{l}708 \\
912\end{array}$ & $\begin{array}{l}850 \\
842\end{array}$ & $\begin{array}{l}231 \\
646\end{array}$ & $\begin{array}{l}231 \\
646\end{array}$ & $\begin{array}{l}294 \\
842\end{array}$ \\
\hline 3 & 787 & 971 & 1022 & 927 & 384 & 659 & 659 \\
\hline 5 & 1071 & 1059 & 1189 & 1106 & 846 & 846 & 1016 \\
\hline 6 & 940 & 1282 & 1329 & 1183 & 585 & 585 & 1039 \\
\hline 9 & 1028 & 998 & 1674 & 1233 & 505 & 505 & 1123 \\
\hline 10 & 423 & 837 & 783 & 681 & 209 & 209 & 209 \\
\hline 12 & 955 & 888 & 1256 & 1033 & 539 & 539 & 848 \\
\hline 13 & 404 & 457 & 722 & 528 & 249 & 249 & 539 \\
\hline 14 & 757 & 1496 & 1087 & 1113 & 242 & 342 & 342 \\
\hline 15 & 1096 & 825 & 1280 & 1067 & 620 & 620 & 932 \\
\hline 16 & 701 & 1067 & 1017 & 928 & 341 & 341 & 527 \\
\hline 17 & 989 & 832 & 781 & 867 & 528 & 528 & 528 \\
\hline 18 & 1067 & 994 & 1393 & 1151 & 701 & 806 & 806 \\
\hline 19 & 882 & 1162 & 1196 & 1080 & 433 & 884 & 955 \\
\hline 20 & 658 & 951 & 776 & 795 & 357 & 570 & 570 \\
\hline 21 & 862 & 768 & 913 & 848 & 152 & 152 & 545 \\
\hline 22 & 366 & 1015 & 970 & 784 & 194 & 475 & 585 \\
\hline 24 & 738 & 871 & 716 & 775 & 439 & 439 & 439 \\
\hline
\end{tabular}

Table 6. Mean legibility distance ( $f t$ ) by site and sign brightness level.

\begin{tabular}{lcccc} 
& \multicolumn{4}{c}{ Sign Luminance } \\
\cline { 2 - 5 } Site & Low & Medium & \multicolumn{1}{l}{ High } & Combined \\
\cline { 2 - 5 } 1 & 201 & 218 & 194 & 204 \\
2 & 226 & 189 & 290 & 235 \\
3 & 107 & 201 & 210 & 173 \\
5 & 170 & 191 & 304 & 222 \\
6 & 132 & 201 & 266 & 200 \\
9 & 176 & 168 & 261 & 202 \\
10 & 123 & 176 & 224 & 174 \\
12 & 184 & 175 & 210 & 190 \\
13 & 133 & 197 & 214 & 181 \\
14 & 151 & 215 & 176 & 181 \\
15 & 208 & 171 & 233 & 204 \\
16 & 67 & 169 & 262 & 166 \\
17 & 195 & 237 & 179 & 204 \\
18 & 198 & 156 & 235 & 196 \\
19 & 97 & 246 & 241 & 195 \\
20 & 161 & 233 & 167 & 187 \\
21 & 225 & 200 & 258 & 228 \\
22 & 103 & 192 & 190 & 162 \\
24 & 210 & 256 & 191 & 219
\end{tabular}

Note: $1 \mathrm{ft}=.3 \mathrm{~m}$. 


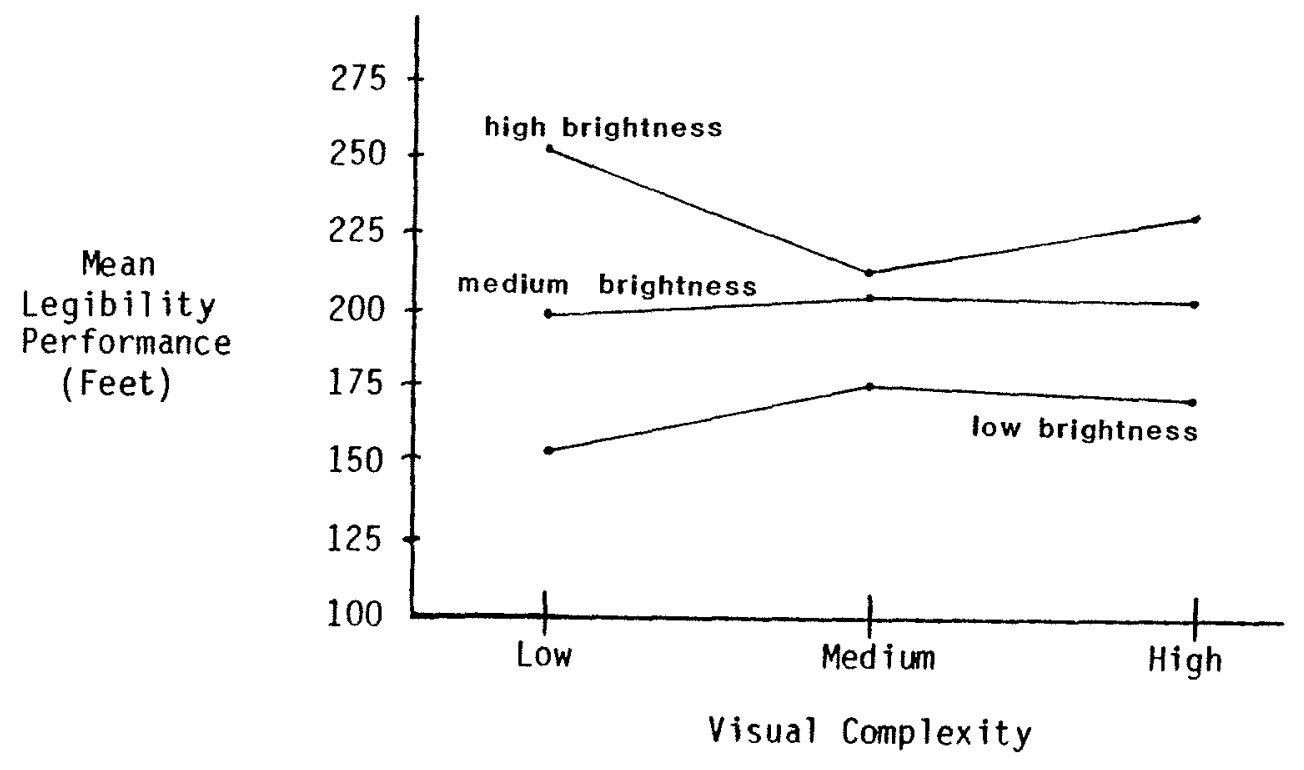

Figure 3. Legibility performance by visual complexity and sign brightness.

Note: $1 \mathrm{ft}=.3 \mathrm{~m}$.

Table 8. Observed mean recognition distances ( $f t)$.

Sign

\begin{tabular}{|c|c|c|c|c|}
\hline \multirow{2}{*}{$\begin{array}{c}\text { Sign } \\
\text { Brightness } \\
\end{array}$} & \multicolumn{4}{|c|}{ Complexity } \\
\hline & Low & Medium & $\mathrm{High}$ & Combined \\
\hline Lo & 979.7 & 943.3 & 592.6 & 838.5 \\
\hline edium & 1109.5 & 1069.2 & 744.4 & 974.3 \\
\hline High & 1398.4 & 1078.7 & 783.6 & 1086.9 \\
\hline Comb & 1162.5 & 1030.4 & 706.8 & 966.6 \\
\hline
\end{tabular}

Note: $1 \mathrm{ft}=.3 \mathrm{~m}$.

Table 9 shows the mean legibility distance for the 3 by 3 factorial of sign brightness and visual complexity. Figure 3 provides a graphic plot of these means.

Table 9. Observed mean legibility distances ( $f t$ ).

Sign Visual Complexity

\begin{tabular}{|c|c|c|c|c|}
\hline \multirow{2}{*}{ Brightness } & \\
\hline & Low & Medium & High & Combined \\
\hline & & & & \\
\hline & 193.2 & 7 & & 20 \\
\hline & .2 & 217.8 & & 233.0 \\
\hline ined & 198.5 & 199.2 & 202.8 & 200.2 \\
\hline
\end{tabular}

Note: $1 \mathrm{ft}=.3 \mathrm{~m}$.

An analysis of variance indicated that the effect of sign brightness was statistically significant ( $\mathrm{p}<.0001)$. As can be seen in table 9, however, there was virtually no influence of visual complex- ity and no interaction of the 2 variables. This is not unexpected, since there is nothing to suggest that visual complexity would influence legibility.

In order to fully and meaningfully evaluate the performance of signs of different brightness levels at different sites, some estimate of required recognition and legibility distance is needed. Perchonok and Pollack (1981) modified the hazard avoidance model of McGee, Moore, Knapp, and Sanders (1978), based upon the concept of decision sight distance, to arrive at sign recognition and legibility distance requirements. The model includes components for detection, reading, decision making, driver response, and vehicle maneuver. Reading time will vary with the amount of information on a sign, decision time varies with decision complexity, and vehicle maneuvering time varies with type of maneuver, the amount of deceleration required, or the density of traffic for lane changes and merging.

To estimate requirements for the yellow diamond warning sign, the shortest reading time requirement ( 1 second) was used, since most of these signs contain symbols or very brief, one word messages. A medium level of decision complexity (2.5 seconds) was used, which may be conservative for this type of sign. The required recognition times and leqibility times were determined for a sign requiring no maneuver (e.g.. Deer Crossing) and a sign requiring a complete stop (very conser- 
tive for this type of sign. The required recognition times and legibility times were determined for a sign requiring no maneuver (e.g., Deer Crossing) and a sign requiring a complete stop (very conservative, since yellow diamond signs never require a complete stop). These time estimates were then converted to the required distances shown in table 10 at different assumed approach speeds. 8

Table 10. Required recognition and legibility distances ( $f t$ ).

\begin{tabular}{|c|c|c|c|c|}
\hline \multirow[b]{2}{*}{$\mathrm{mph}$} & \multicolumn{2}{|c|}{$\begin{array}{c}\text { Recognition } \\
\text { Distance }\end{array}$} & \multicolumn{2}{|c|}{$\begin{array}{l}\text { Legibility } \\
\text { Distance }\end{array}$} \\
\hline & $\begin{array}{c}\text { No } \\
\text { Mane uver }\end{array}$ & Stop & $\begin{array}{c}\text { No } \\
\text { Maneuver }\end{array}$ & Stop \\
\hline $\begin{array}{l}35 \\
45 \\
55\end{array}$ & $\begin{array}{l}325 \\
419 \\
520\end{array}$ & $\begin{array}{l}489 \\
691 \\
927\end{array}$ & $\begin{array}{l}179 \\
231 \\
290\end{array}$ & $\begin{array}{l}343 \\
503 \\
697\end{array}$ \\
\hline te: & $1 \mathrm{mi} / \mathrm{h}=$ & $1.6 \mathrm{k}$ & $1 \mathrm{ft}=$ & $\mathrm{m}$. \\
\hline
\end{tabular}

Since yellow warning signs typically do not demand an extreme deceleration prior to the location of the sign, the distances required for the no maneuver condition are most relevant. Comparing the required distances of table 10 with the observed mean distances of tables 8 and 9 indicates areas of concern. The 520-ft $(156-m)$ recognition distance required at $55 \mathrm{mi} / \mathrm{h}(88 \mathrm{~km} / \mathrm{h})$ can easily be met by the most degraded sign in low complexity situations. However, in high complexity situations, the average performance of the most degraded signs was barely above the reguired distance for $55 \mathrm{mi} / \mathrm{h}(88$ $\mathrm{km} / \mathrm{h})$. Since mean performance does not allow a sufficient margin of safety, signs with Type II sheeting should not be allowed to degrade below 36 SIA (72 percent of Federal specifications) in high complexity locations.

Legibility may present even more of a problem. In an absolute sense it would seem that the 167-ft (50.1-m) (table 9) average legibility of the low brightness sign would be inadequate in all situations. Since only $5-$ in $(12.5-\mathrm{cm})$ letters were used, the degraded sign had legibility of approximately $30 \mathrm{ft} / \mathrm{in}(3.6 \mathrm{~m} /$ $\mathrm{cm}$ ), while the medium brightness and high brightness signs had $40 \mathrm{ft} /$ in $(4.8 \mathrm{~m} / \mathrm{cm})$.
However, since many yellow warning signs contain symbols, legibility of $30 \mathrm{ft} /$ in $(3.6 \mathrm{~m} / \mathrm{cm})$ may provide adequate distance, particularly when either no maneuver or only a modest deceleration is necessary.

Since mean values do not allow a sufficient margin of safety, the 100 th percentile is perhaps a better index in examining minimum driver requirements. Table 5 includes the $100 t h$ percentile recognition performances for all of the sites. It can be seen that 8 sites $(2,5,6,9,12$, 15,17 , and 18 ) showed a 100 th percentile recognition distance greater than $500 \mathrm{ft}$ $(150 \mathrm{~m})$ when signs deqraded to 36 percent of Federal specification (lowest brightness) were included. This is well in excess of the required distances when no vehicle maneuver is required and sufficient to satisfy the requirement of a complete stop from approach speeds up to $45 \mathrm{mi} / \mathrm{h}(72 \mathrm{~km} / \mathrm{h})$. On the basis of recognition performance, these 8 sites were said to be low complexity. Also in table 5 we see 4 sites $(1,10,13$, and 21) had 100 th percentile recognition performance for medium and high brightness signs below the minimum value of 325 ft $(97.5 \mathrm{~m})$ for no maneuver at $35 \mathrm{mi} / \mathrm{h}$ $(56 \mathrm{~km} / \mathrm{h})$. Four other sites $(14,16,22$, and 24 ) failed to provide adequate recognition distance with approach speeds to $55 \mathrm{mi} / \mathrm{h}(88 \mathrm{~km} / \mathrm{h})$ without a maneuver required. On the basis of performance these 8 sites were said to be high complexity. The 100 th percentile performance for half of these 8 sites was raised above the required distance for $55 \mathrm{mi} / \mathrm{h}$ $(88 \mathrm{~km} / \mathrm{h})$ approach speeds when the medium brightness signs were included. Since luminance deterioration is a natural occurrence and performance remained substandard at 4 sites with non-deteriorated Type II signs, the need for Type III (high intensity) sheeting seems to be indicated.

Decision Rules for Luminance Requirements

To evaluate the practical implications of this work, it is helpful to use the classification of errors in hypothesis testing. A false negative is the acceptance of the alternative hypothesis when it is false, and a false positive is failing to accept the alternative hypothesis when it is true. Changing the

\footnotetext{
8 The recognition distances include distances for reading, decision making, driver response, and vehicle maneuver, but omit the distance associated with detection, since the performance criterion observed in the field study represented recognition of the sign as a yellow diamond, implying detection had already occurred. The legibility distances include distances for decision making, driver response, and vehicle maneuver, but omit the distances associated with detection and reading, since the performance criterion observed in the field represented the distance after legibility.
} 
decision rule increases one type of error and decreases the other. The greater the reliability of measurement, the easier it is to minimize both types of errors, but because of the error in both the measurement of complexity and luminance, classifying sites on complexity to specify luminance needs is a process which must tolerate a good deal of error. Since safety is an issue as well as cost, care must be taken to minimize errors which may create hazards. To make a decision as to whether a site is low complexity and can perform adequately with a sign of poor l uminance, one must accept a high false positive rate (classify sites as not low complexity which perhaps are low). Likewise, in deciding whether a site has high complexity and requires special attention with regard to sign I uminance, one must accept a high false negative rate (classify sites as high complexity which maybe are not).

The first step in finding a decision rule to implement these strategies is to categorize the sites as low or high complexity based upon the empirically obtained recognition distance. Using the required recognition distances derived from the hazard avoidance model (McGee et al., 1978; Perchonok \& Pollack, 1981) which are shown in table 11, we decided to classify sites as low complexity if their 100 th percentile recognition distance was greater than $500 \mathrm{ft}(150 \mathrm{~m})$ across all sign brightness levels. In other words, for a site to be classified low complexity, every driver had to recognize the sign more than $500 \mathrm{ft}(150$ m) away, even if it were degraded to only 36 percent of the Federal standard. High complexity sites were defined as sites which had a 100 th percentile recognition distance below $500 \mathrm{ft}(150 \mathrm{~m})$, excluding the most degraded signs. In other words, if only one driver failed to recognize a new sign or a sign degraded to 72 percent of Federal specification (medium brightness) at less than $500 \mathrm{ft}(150 \mathrm{~m})$, the site was considered of high complexity. Sites classified either low or high complexity are identified by their looth percentile recognition distance in column A of tables 11 to 14 .

The next step in the search for a decision rule was to compute a predicted recognition distance using each of the candidate regression equations presented earlier. While the reliability of the scales has been addressed, the validity of the regression equations has not. The proper way to address this issue is to cross validate using another sample of sign locations. The regression equations would be used to predict performance at the new site, and the correlation of the observed and predicted performance would be a measure of the equations' true validity. An estimate of what this true validity might be can be obtained from figures given by Ezekiel and Fox (1963). Given samples of 19 sites and the observed multiple $R^{\prime} s$ of .75 to .81 for 4 variable equations, 1 in 20 samples would give multiple $R^{\prime} s$ this high even if the true correlation were only in the .4 to .5 range.

The 2 equations tested (equations 1 and 2) both use 4 scales. The predicted recognition distance (visual complexity) using each equation is given in column $B$ of tables 11 to 14 . The 2 equations were the 2 with the highest validities using 4 scales. They have 2 of the 4 variables in common. Five-, 6-, and 8-variable equations were evaluated, but resulted in little or no change from the results which are presented in tables 11 to 14 .

The third step was to locate the cutoff scores which would eliminate the hazardous errors. These were the highest predicted score of the sites not classified low complexity (column $\mathrm{C}$, tables 11 and 12) and the highest predicted score of the sites classified high complexity (column $C$, tables 13 and 14). With the column $C$ heading used as a cutoff score for site classification, erroneous classifications were identified.

With respect to low complexity, false positives are sites not identified as being of low complexity, but which on the basis of the empirical data are of low complexity (in columns C, D, and $E$ of tables 11 and 12). False negatives are sites which are classsified as being of low complexity, but which are considered not of low complexity based upon the empirical data. The cutoff score in column $C$ was chosen to ensure that there would be no false negatives, i.e., sites classified of low complexity which are not. To get zero false negatives one has to tolerate false positives with either equation. Six of the 8 low complexity sites will be misidentified. If we are willing to tolerate 1 false negative in the 11 sites which are considered not low complexity, we can use equation 1 to reduce the false positives to 5 of the 8 low complexity sites. Decreasing the cutoff score increases the false negatives. A cutoff score of 1078 with equation 1 appears best for identifying low complexity sites. This cutoff score identified almost half of the low complexity sites, significant for potential savings in sign replacement costs, and 
Table 11. Evaluation of 3 cutoff scores for identifying 8 low complexity sites using regression equation 2 .

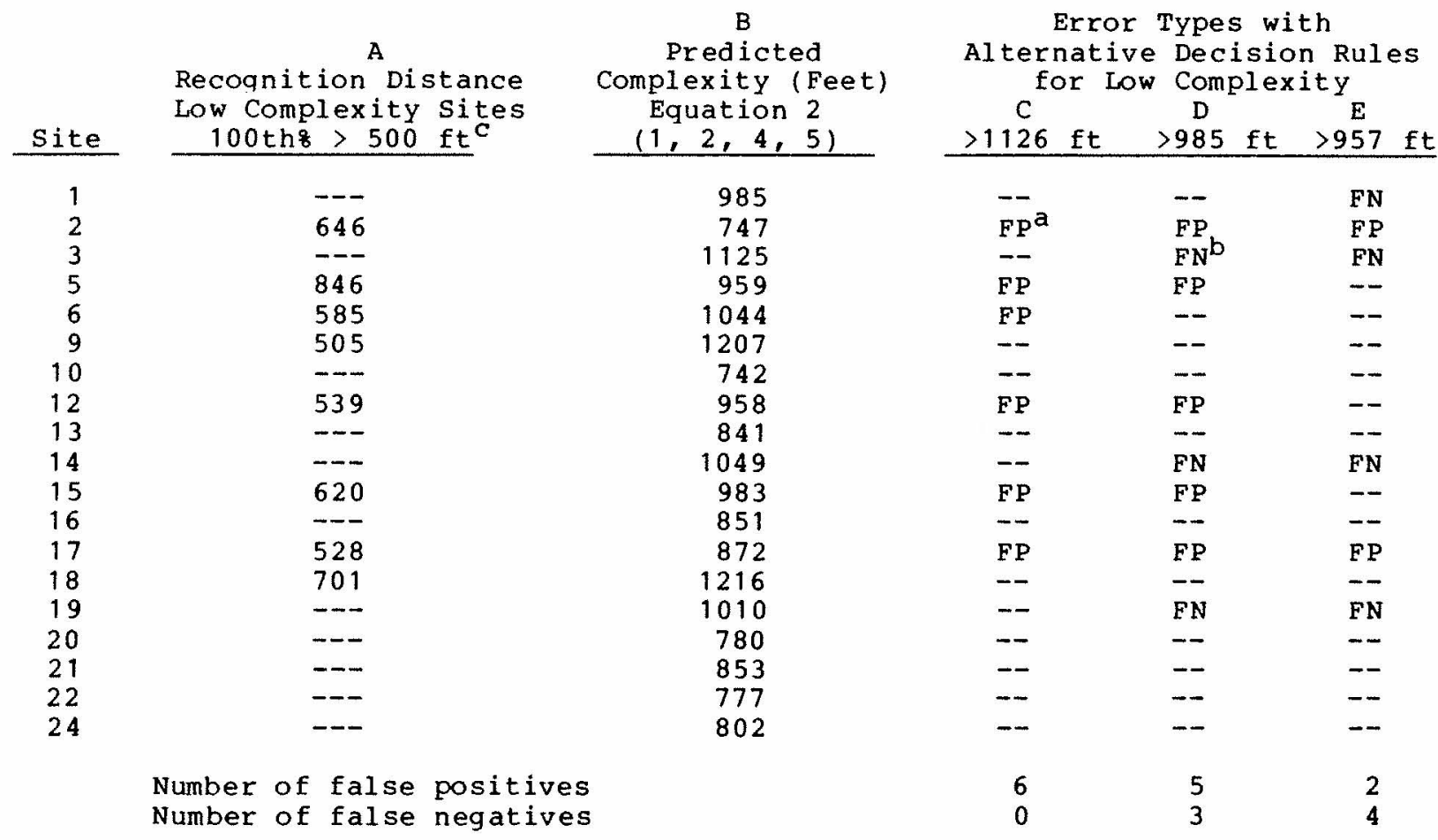

Table 12. Evaluation of 3 cutoff scores for identifying 8 low complexity sites using regression equation 1 .

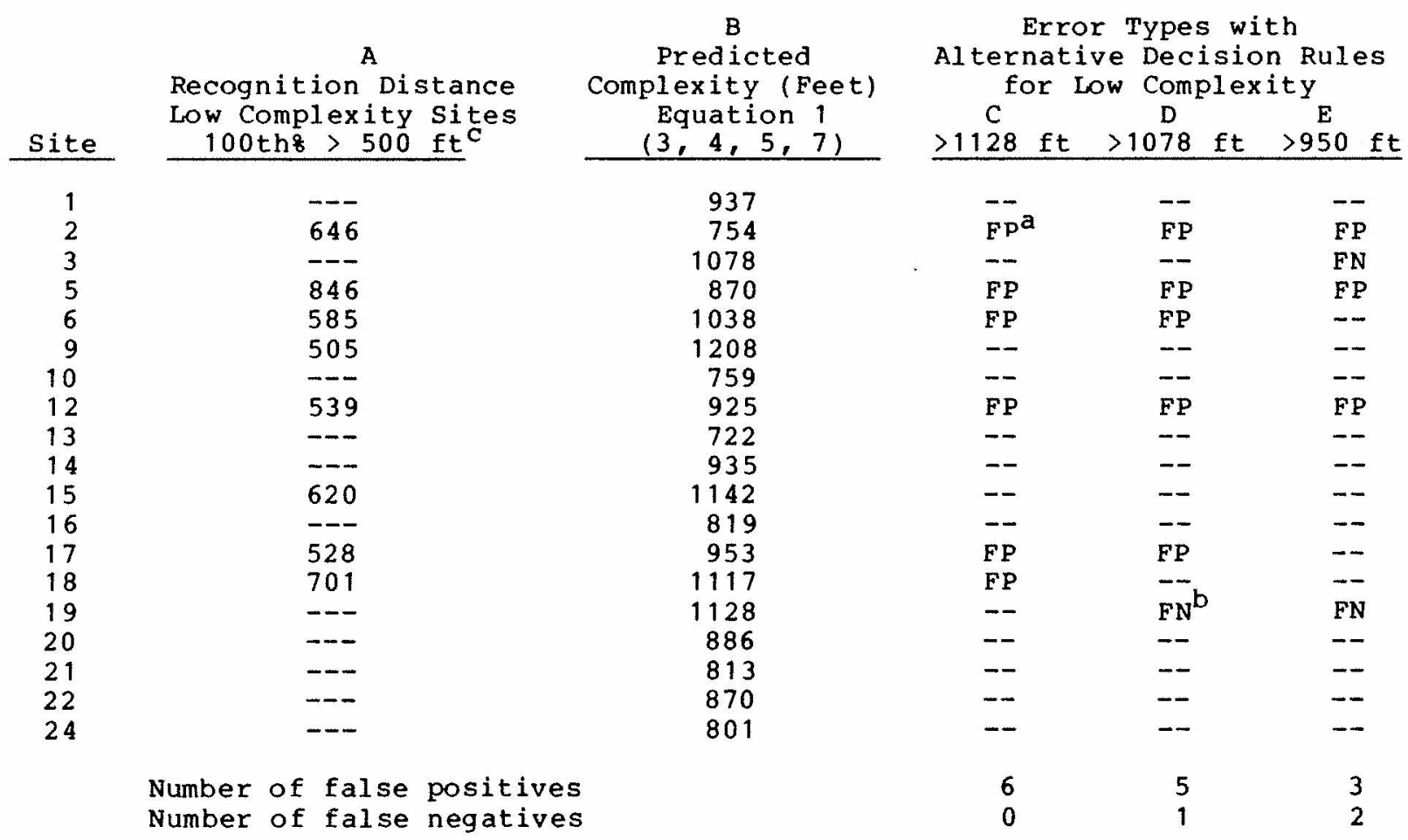

Note: $1 \mathrm{ft}=.3 \mathrm{~m}$.

a $F P$ false positive.

b $F N=$ false negative.

c looth percentile score for all sign luminances. 
Table 13. Evaluation of 3 cutoff scores for identifying 8 high complexity sites using regression equation 2 .

\begin{tabular}{|c|c|c|c|c|c|c|c|c|}
\hline Site & $\begin{array}{c}\text { A } \\
\text { Recognition Distance } \\
\text { High Complexity Sites } \\
100 \text { th } 8<500 \mathrm{ft}^{\mathrm{C}}\end{array}$ & $\begin{array}{c}\text { B } \\
\text { Predicted } \\
\text { Complexity (Feet) } \\
\text { Equation } 2 \\
(1,2,4,5) \\
\end{array}$ & \multicolumn{6}{|c|}{$\begin{array}{l}\text { Error Types with } \\
\text { Alternative Decision Rules } \\
\text { for High Complexity } \\
\text { C } \quad \text { D } \\
>1050 \mathrm{ft} \quad>986 \mathrm{ft}>854 \mathrm{ft}\end{array}$} \\
\hline 1 & 231 & 985 & -- & & - & & FP & \\
\hline 2 & --- & 747 & $\mathrm{FN}^{\mathrm{a}}$ & & FN & & FN & \\
\hline 3 & --- & 1125 & -- & & -- & & -- & \\
\hline 5 & --- & 959 & $\mathrm{FN}$ & & FN & & -- & \\
\hline 6 & --- & 1044 & FN & & - & & -- & \\
\hline 9 & --- & 1207 & - & & - & & -- & \\
\hline 10 & 209 & 742 & -- & & -- & & -- & \\
\hline 12 & -- & 958 & FN & & FN & & -- & \\
\hline 13 & 249 & 841 & -- & & -- & & -- & \\
\hline 14 & 342 & 1049 & -- & & FP $^{\mathrm{b}}$ & & FP & \\
\hline 15 & -- & 983 & FN & & FN & & -- & \\
\hline 16 & 341 & 851 & -- & & -- & & -- & \\
\hline 17 & -- & 872 & $\mathbf{F N}$ & & FN & & -- & \\
\hline 18 & -- & 1216 & -- & & -- & & -- & \\
\hline 19 & --- & 1010 & FN & & -- & & -- & \\
\hline 20 & --- & 780 & FN & & FN & & FN & \\
\hline 21 & 152 & 853 & -- & & - & & - & \\
\hline 22 & 475 & 777 & -- & & -- & & -- & \\
\hline 24 & 439 & 802 & -- & & -- & & -- & \\
\hline & $\begin{array}{l}\text { er of false positives } \\
\text { er of false negatives }\end{array}$ & & $\begin{array}{l}0 \\
8\end{array}$ & & 1 & & 2 & \\
\hline
\end{tabular}

Table 14. Evaluation of 3 cutoff scores for identifying 8 high complexity sites using regression equation 1 .

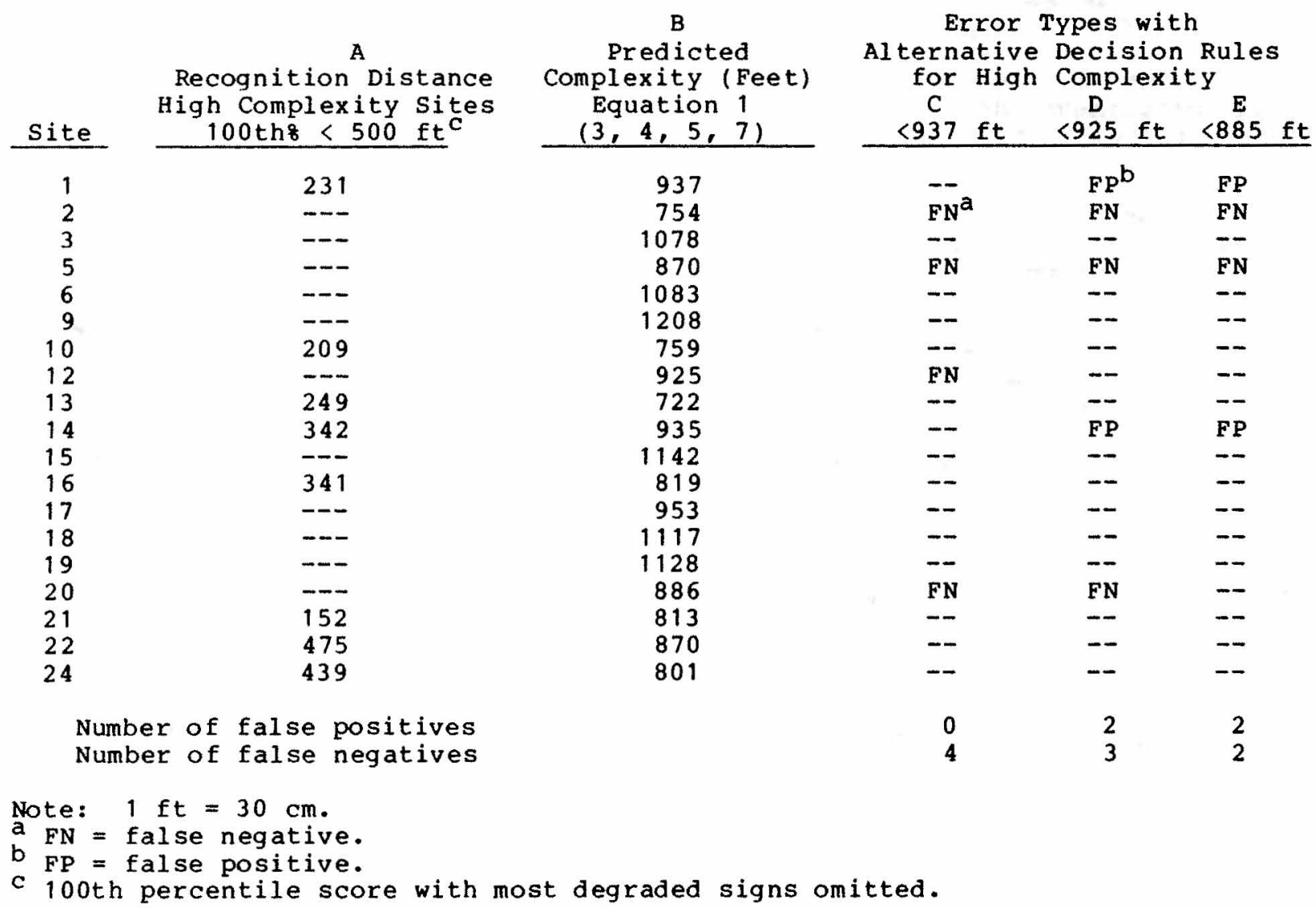


missed only one 9 of 11 sites which should not be considered low complexity.

With respect to high complexity, false negatives are sites classified as being of high complexity, but which on the basis of the empirical data were not of high complexity (in columns C, D, and $E$ of tables 13 and 14 ). False positives are sites which were not classified as being of high complexity, but which on the basis of the empirical data are high complexity sites. To get the false positive rate to zero, one must tolerate 8 false negatives with equation 2 and 4 false negatives with equation 1 . In other words, with equation 2 we would misidentify 8 of 11 sites as high complexity, and only 4 would be misidentified with equation 1 . Accepting 2 false positives with equation 1 does not give enough improvement in the false negative rate to make the potential increase in risk acceptable. With equation 2 however, the tradeoff might be worthwhile. Accepting 2 false positives in the 8 high complexity sites results in a reduction of the false negative rate from 8 of 11 to 2 of 11 .

\section{F. Conclusions}

The main effects of the independent variables of sign brightness and visual complexity were consistent with hypotheses suggested by earlier research (Mace et al., 1982). In general visual complexity has a negative effect on sign recognition, but no effect on legibility; while sign brightness has a positive effect on both recognition and legibility.

Further support was obtained for the general hypothesis that sign brightness can offset the detrimental effects of $v$ isual complexity. The data suggest specifically that at low complexity sites, Type I y yellow sheeting degraded to 36 percent of Federal specification is adequate for the types of situations studied. At high complexity sites, Type II yellow sheeting should not be allowed to degrade below the 50 percent Federal specification for accelerated weathering, and perhaps should be replaced when it reaches the 72 percent ( 36 SIA) value of the medium-degraded signs studied. Only when complexity is high and either the approach speed is high or a lane change or extreme deceleration is required might a sign of new Type II sheeting be inadequate used alone, unsupplemented by advanced warning.

It must be remembered that the conclusions with respect to sign reflectivity are restricted to roads without extreme curvature. A sign of 36 percent reflectance which was satisfactory on a straight road in a low complexity location might be inadequate on a road with a severe right curve. To provide results generalizable to conditions other than the straight approach which characterized the Trenton test sites, the following guidelines are offered. Regardless of sign placement or road curvature, yellow diamond warning signs in low complexity areas should have a minimum luminancelo of .14 candelas $/ \mathrm{ft}^{2}\left(1.51 \mathrm{candelas} / \mathrm{m}^{2}\right)$. The same signs in high complexity areas require a minimum of .25 candelas/ $\mathrm{ft}^{2}$ $\left(2.69 \mathrm{candelas} / \mathrm{m}^{2}\right)$. Because of 1 ight scatter these values may double in bright ambient areas.

Within the range of brightness and complexity studied, their were no interactive effects of visual complexity and sign brightness. The absence of an interaction between sign brightness and visual complexity appears contrary to expectations based upon earlier research (Mace et al., 1982), which suggested that brightness and complexity interacted such that sign brightness has its greatest effect on visual peformance at the middle ranges of complexity and that:

- At high levels of complexity brighter reflective signs might not improve performance.

- At low complexity locations even signs of very degraded luminance perform satisfactorily.

With respect to high levels of complexity, either the hypothesis was wrong or the levels of complexity tested in this study were not high enough. It may be that if higher levels of complexity were sampled, recognition distance would drop for all levels of sign brightness to a distance less than legibility distance.

9 The specific site missed was 19. It was not classified low complexity because its $100 \mathrm{th}$ percentile score was $433 \mathrm{ft}(129.9 \mathrm{~m})$ less than the required $500 \mathrm{ft}$ (150 m). However, its $85 \mathrm{th}$ percentile score was $884 \mathrm{ft}(265.2 \mathrm{~m})$, suggesting that this one false negative might not be too serious.

10 Measured at $500 \mathrm{ft}(105 \mathrm{~m})$ from the sign with a photometer calibrated for Iuminance at the 3.18 candelas $/ \mathrm{ft}^{2}$ level $\left(34.26\right.$ candelas $\left./ \mathrm{m}^{2}\right)$. 
This is only speculation. At the highest levels of complexity measured in this study sign brightness had a positive effect, and the possibility exists that performance might even be improved by a material with higher specific luminance, such as Type III sheeting.

With respect to low complexity sites, the contradiction with the hypothesis from the earlier study is semantic. We should have hypothesized that at low complexity sites, sign brightness would not affect driver performance. Instead, the hypothesis referred to visual performance. The hypothesis was generated by a field study which used speed reduction in response to a SPEED TRAP sign as the dependent variable. The visual performance of the drivers was not measured. If driver performance and not recognition distance had been measured in the present study, it is unlikely that any difference would have been observed at the low complexity sites because sign recognition distances for all levels of brightness are far in excess of what drivers require. It is also probably true that there would have been no performance difference at medium complexity sites either. Only at the high complexity sites is it likely that a performance difference would have been observed, because at these sites recognition distances dropped to levels which in some cases were less than recommended requirements.

The results further suggest that a simple and useful procedure for measuring visual complexity is possible. The scales tested produced very high reliability when used in the field. The validity is more suspect since crossvalidation was not performed. However, using tables provided by Ezekiel and Fox (1963) based upon Fisher's methods for determining the reliability of observed correlations, we can say that the true correlation for the multiple correlations presented is greater than .5, and that this statement will be wrong in only 1 out of 20 samples. This seems sufficient to suggest utility for the recommended procedure documented in Chapter $\mathrm{V}$.

\section{A PROCEDURE FOR CATEGORIZING VISUAL COMPLEXITY}

The purpose of this chapter is to provide specific directions in using a simple procedure to classify highway locations with respect to their nighttime visual complexity.

Many things influence the visibility of a sign, but one of the most important is the visual complexity of the sign location. One may imagine 2 signs of equal size and brightness. The first is mounted on the shoulder of a rural road that cuts through a forest, and a driver is approaching it on a dark night with lights on high beam. Almost any sign will be visible under these conditions. The second sign is mounted at the corner of Broadway and 4 2nd street in New York City. Many things will attract the driver's attention: signs, lights, and detail all around. In the city, the sign had better be very big and very bright, or it will be lost in the visual "noise." Obviously these 2 cases are extremes, but background complexity doesn't have to be extreme to have an effect.

The procedure to classify sign locations with respect to their visual complexity was developed as part of research on sign luminance requirements sponsored by the Federal Highway Administration. This research has shown that when visual complexity is low, signs with reflectivity less than 50 percent of Federal specifications for Type II sheeting (engineering grade) provide adequate recognition and legibility distance. However, the research also suggested that new signs of Type II sheeting may not provide adequate I uminance at high complexity locations. Therefore, before examining the details of the procedure, the reader should have a good understanding of its intended application as well as some understanding of its 1 imitations.

The recommended procedure is not intended to encourage sign neglect; rather it is offered as a tool to be used in sign maintenance management. Because of Iimited budgets, signs which ought to be replaced are not, and often sign inspections cannot be performed because of limited personnel resources. If locations with a great deal of visual complexity were identified, signs located at these sites could be given priority in any sign inspection budget. Similarly, low complexity locations could be given low priority.

The usefulness of classifying the visual complexity of sign locations will increase as automated sign inventories become prevalent. By making the complexity categorization part of the sign inventory record, sign maintenance managers can easily make software responsive to this dimension in any inspection or replacement program. A manager might tell the computer what he considers the most critical signs and receive a report 1 isting all high complexity locations with such signs, including their last maintenance and inspection dates. Similarly, a 
manager might request that a computer be programmed to generate work orders, and to withhold signs designated least critical where visual complexity is low. In that way, more critical signs at high complexity locations could be given priority in a limited budget.

The procedure to be described doesn't depend on the type of sign or sign location to be evaluated. However, anyone choosing to use the procedure should be aware that the procedure was developed using only yellow diamond warning signs mounted on the right shoulder of the road. Also, the procedure has not been cross-validated. The lack of cross-validation means that subsequent study might result in variations and improvement.

Since the scales did have a high degree of reliability, it is suggested that the recommended procedure is the best available technique for evaluating complexity. with respect to right shoulder yellow warning signs, it is also a legitimate and useful sign maintenance management tool. The generalizability of the tool to other locations and sign colors is less certain. The procedure would probably have validity at other locations; but since the yellow diamond is usually installed on the right shoulder, this has no consequence without color generalizability. Since evidence exists that sign luminance, color, and shape interact with complexity, the procedure cannot be suggested for use with other sign colors and shapes at this time. If the utility of the procedure to be described becomes widely recognized, the research necessary to provide similar procedures for other signs and locations will likely be undertaken.

The procedure for classifying sites on $v$ isual complexity is to have 1 or 2 people visit the site after dark under normal traffic conditions and rate the location at approximately $500 \mathrm{ft}(150 \mathrm{~m})$ from sign placement using the 4 scales described on the Visual Complexity Rating Form, figure 5. The scale values 11 chosen are simply added, and the resulting number is the site complexity value. For example, suppose a location is given the following values on the 4 complexity scales in figure 5 .

$\begin{array}{rr}\text { scale 1 } & 13 \\ \text { scale 2 } & 8.5 \\ \text { scale 3 } & -11.5 \\ \text { scale 4 } & -1 \\ \text { visual complexity }= & 9\end{array}$

The score obtained is used to classify the site in the following way. When the site complexity value is greater than 11 , the site should be classified low complexity, and if the score is less than 9.5 , the site should be classified high complexity. Two photographs (figure 4) illustrate the scaling procedure. 12

Skillful evaluation of a site is a matter of asking the questions associated with each scale, and answering them systematically based on observation. The questions should be considered independently. A preceding question should not influence a response to a later one. After a response to each question (scale) is made, the corresponding scale value to the left should be circled. The complexity rating is the sum of the 4 scale values. The terms "scene" and "cone" used in the scale questions are defined below:

- Scene - The "scene" is everything one sees when looking at a site through the windshield of a vehicle. It includes the road, the sky, and everything within sight on both sides of the road.

- Cone - The "cone" is the portion of the right side of the road where one would normally look for traffic signs. It can extend from a few feet in front of the vehicle to the horizon, but when driving, most people monitor an area in the cone from $200 \mathrm{ft}(60 \mathrm{~m})$ to $600 \mathrm{ft}(180 \mathrm{~m})$ down the road. The cone may include traffic signs, advertising signs, traffic lights, street lights, and even store fronts if they are near the road.

11 The scale values are derived from equation 1 in chapter IV. All numbers including the cutoff score have been reduced by a factor of 100 , and the constant has been removed from consideration by adding 5 to every scale value.

12 The scale values shown under the 2 scenes represent mean responses on the 4 scales of the Visual Complexity Rating Form that were obtained from subjects who rated the scenes in the field as described in chapter IV of this report. Scale values from the field raters are shown because scores obtained from raters of photographs lacked reliability as described in chapter IV. The photos are used here only to illustrate the scenes. 


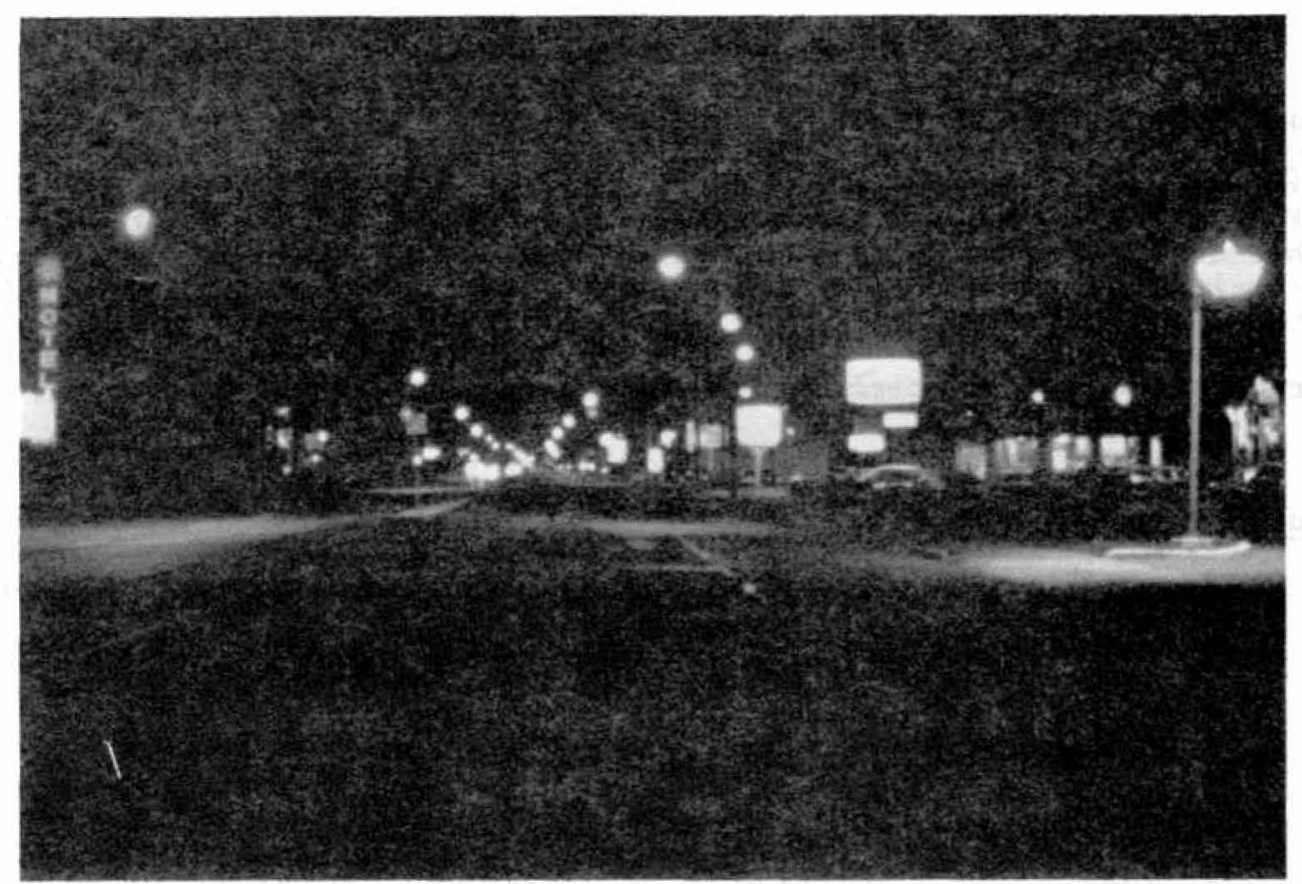

Scene 21

\begin{tabular}{|c|c|c|c|c|c|}
\cline { 2 - 6 } & Scale 1 & Scale 2 & Scale 3 & Scale 4 & Complexity Value \\
\hline Value & 21 & 19 & -17 & -13 & 10 \\
\hline
\end{tabular}

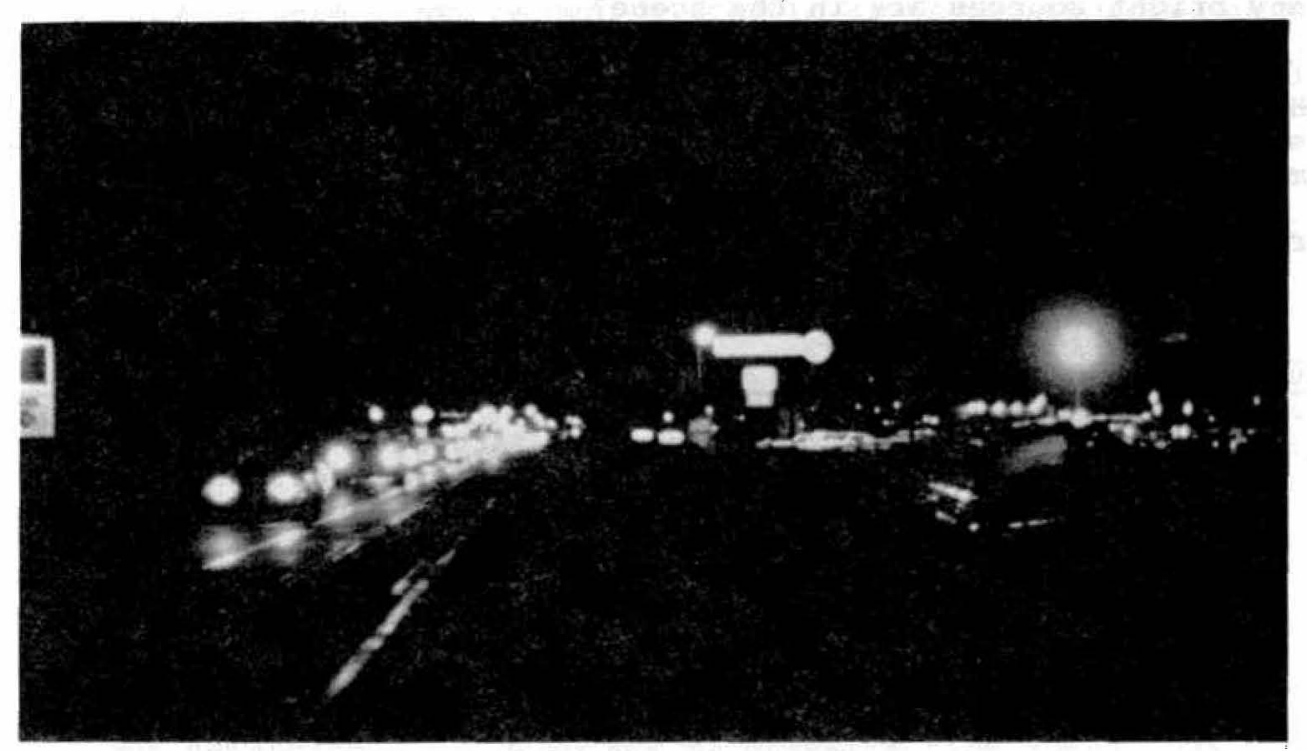

Scene 15

\begin{tabular}{|c|c|c|c|c|c|}
\cline { 2 - 6 } \multicolumn{1}{c|}{} & Scale 1 & Scale 2 & Scale 3 & Scale 4 & Complexity Value \\
\hline Value & 17 & 19 & -11.5 & -13 & 11.5 \\
\hline
\end{tabular}

Figure 4. Example of complexity scaling. 


\section{How much detail is visible in the scene?}

Detail is anything you can see against the darkness. It includes lights and objects that the lights illuminate. Consider the entire field of view including the road, the horizon, and the area on both sides of the road. Can many objects be picked out?

Answer this question by circling the appropriate scale value:

\begin{tabular}{|c|c|c|}
\hline Scale Value & $\begin{array}{l}\text { Subjective } \\
\text { Description }\end{array}$ & Scale Definition \\
\hline 25 & $\begin{array}{l}\text { A great deal } \\
\text { of detail }\end{array}$ & $80 \%$ or more of the scene has visible detail \\
\hline 21 & $\begin{array}{l}\text { A lot of } \\
\text { detail }\end{array}$ & 60 to 808 of the scene has visible detail \\
\hline 17 & Average & 40 to 608 of the scene has visible detail \\
\hline 13 & Little detail & 20 to 408 of the scene has visible detail \\
\hline 9 & $\begin{array}{l}\text { Very little } \\
\text { detail }\end{array}$ & $\begin{array}{l}208 \text { or less of the scene has visible detail (dark } \\
\text { country road) }\end{array}$ \\
\hline
\end{tabular}

2. How many bright sources are in the scene?

Are there many bright spots -- streetlights, internally lighted signs, bright billboards, car lights, parking lot lights, lighted store windows, and bright reflections from glass and metal?

Answer this question by circling the appropriate scale value:

Scale Value

22.5

19

15.5

12

8.5
Subjective Description

Very many

Many

Average

Few

very few

\section{Scale Definition}

The scene is saturated with bright sources (many strong lights and reflections, bright signs, bright store fronts, and car lights)

Above average

Moderate

Some distant lights

Virtually no bright sources

Figure 5. Visual complexity rating form. 
3. How much detail is visible in the cone?

Are many lights and objects visible?

NOTE: Much detail may be visible in the scene, but very little in the cone. For example, a bridge approach may be lighted to the degree where you can see pavement seams. The horizon may be cluttered with lights. The cone, however, can include just a barely discernible bridge railing. Conversely, a dark country road with a lighted service station in the cone may exhibit little scene detail, but above average cone detail.

Answer this question by circling the appropriate scale value:

\begin{tabular}{|c|c|}
\hline Scale value & $\begin{array}{l}\text { Subjective } \\
\text { Description }\end{array}$ \\
\hline-22.5 & $\begin{array}{l}\text { A great deal } \\
\text { of detail }\end{array}$ \\
\hline-17 & A lot of detail \\
\hline-11.5 & Average \\
\hline-6 & Little detail \\
\hline-0.5 & $\begin{array}{l}\text { Very little } \\
\text { detail }\end{array}$ \\
\hline
\end{tabular}

Scale Definition

808 or more of the cone has visible detail (city area with many lights and objects in the cone)

60 to $80 \%$ of the cone has visible detail

40 to 608 of the cone has visible detail

20 to 408 of the cone has visible detail

208 or less of the cone has visible detail (dark country road with virtually no illumination in the cone)

\section{How demanding would driving be at this location?}

Drivers' ability to detect and recognize traffic signs deteriorates as the demands of driving increase. The demands increase with the number of lanes and the number of vehicles traveling in the same direction. Many pinpoint lights to the left and right of the cone also increase the demands. Pedestrians and intersections controlled by traffic signals or signs add to the difficulty.

Answer this question by circling the appropriate scale value:

\begin{tabular}{|c|c|}
\hline Scale Value & $\begin{array}{l}\text { Subjective } \\
\text { Description }\end{array}$ \\
\hline-25 & Very demanding \\
\hline-19 & Demanding \\
\hline-13 & Average \\
\hline-7 & Not demanding \\
\hline-1 & Easy \\
\hline
\end{tabular}

Scale Definition

808 or more of driver's time spent looking for driving information -- would not try to light cigarette in this location

70 to 808 of driver's time spent looking for driving information

60 to 708 of driver's time spent looking for driving information

50 to 608 of driver's time spend looking for driving information

$50 \%$ or less of driver's time spent looking for driving information -- no problem lighting cigarette in this location

Figure 5. Visual complexity rating form (continued). 
APPENDIX A. FACTOR ANALYSIS PROCEDURE AND RESULTS

Factor analysis is not an exact analytic tool; that is, it does not produce a single unequivocal result. The available techniques are capable of producing many different solutions, which is the principal reason why factor analysis remains as much art as science. Because of this, factor analysis is more often viewed as an exploratory procedure than a technique for hypothesis testing, although it can be used for both. Its use in this study was strictly exploratory. Our interest was in reducing a large number of variables measured in the previous study (Mace et al., 1982) to a small set of factors which have both theoretical and practical meanings. The greater the conceptual meaning of the factors, the greater the reliability of any attempt to subjectively use them to rate complexity. Because the analysis was exploratory and the results were to be validated in subsequent phases of the study, there was no reason why several different factor-analytic approaches could not be evaluated.

With regard to the correlation matrix, a decision had to be made concerning what variables from the earlier study to include. Since our interest was only with the dimensions of visual complexity, all of the contrast measures were excluded and only the scene and surround measures were retained. Several additional variables were eliminated because of very high correlations with other variables. A list of the 40 variables selected for inclusion in the factor analysis is shown in table 15 .

Several different procedures for factor analysis were employed. The most common methods included principal component analysis and principal factor analysis. Principal component analysis assumes that all of the variance of each variable is common to one or more of the other variables; hence, unity or 1.0 is placed in the diagonal of the correlation matrix. Principal factor analysis assumes that some of the variables have specific variance and therefore a portion of their variance is uncorrelated with other variables. Under this assumption $\mathrm{R}^{2}$ (the square of the multiple correlation of each variable with all other variables) was placed in the diagonal. There was little difference in the solutions obtained by these methods, and the principal factor analysis was retained.

Three different orthogonal rotations were tested (varimax, quartimax, and equivax) as was an oblique rotation. Again the results were not substantially different. The loadings of the varimax rotation are shown in table 15. Although some solutions had as many as 8 factors and the one shown in table 15 only 5 factors, and although the differences in factor loadings were in some cases substantial, there was general agreement among all of the solutions in the conceptual interpretation of at least 4 of the 5 factors. The principal factor analysis with varimax rotation was selected because it gave the simplest and most conceptually interpretable solution.

The process of developing subjective scales from the output of factor analysis was anything but unequivocal. Different researchers might look at the variables and factor loadings contained in table 15 and arrive at different cognitive interpretations of the dominant underlying dimensions. Our assessment of these dimensions is summarized in the following factor designations:

- Ambiguity of Road Orientation.

- Presence of Distracting Visual objects.

- Presence of Other Traffic Signs.

- Uniformity and Brightness in the Cone.

- Heavy Demand fron Driving Task.

The descriptions of each of these factors are contained in chapter III.

APPENDIX B. INSTRUCTIONS FOR COMPLEXITY EVALUATION (FIELD STUDY)

Purpose of the study

Maintaining traffic signs is a problem for every highway agency. Signs deteriorate not only because of vandalism or collisions, but also from age and the effects of weather, sun, traffic, dirt, etc. Deterioration is particularly damaging to the luminance of signs and your ability to detect and read them at night.

While signs must be repaired when obvious damage occurs, the need for maintenance because of gradual deterioration is not as easily determined. In the first place, some signs are less critical and do not need to be recognized as far away as others. Second, it has been found that the setting in which a sign is placed significantly affects how quickly you recognize it. Even severely deteriorated signs may perform adequately in areas where complexity of the background is low. Since many, particularly rural, areas are low in visual complexity, sign inaintenance cost could be reduced if a 
Table 15. Variable factor weights.

\begin{tabular}{|c|c|c|c|c|c|c|}
\hline $\begin{array}{l}\text { Variable } \\
\text { Name }\end{array}$ & Description & $\begin{array}{c}\text { Factor } \\
1\end{array}$ & $\begin{array}{l}\text { Factor } \\
2\end{array}$ & $\begin{array}{c}\text { Factor } \\
3\end{array}$ & $\begin{array}{l}\text { Factor } \\
4\end{array}$ & $\begin{array}{c}\text { Factor } \\
5\end{array}$ \\
\hline \multicolumn{7}{|c|}{ Scene Variables } \\
\hline RORIE & $\begin{array}{l}\text { Road orientation: right } \\
\text { hand edge of road is unambiguous }\end{array}$ & 45 & 13 & 14 & 3 & 6 \\
\hline COGREL & $\begin{array}{l}\text { Cognitive factors } \\
\text { relevant to driving }\end{array}$ & 18 & -24 & 20 & 0 & 31 \\
\hline COGINT & $\begin{array}{l}\text { Cognitive factors; interesting } \\
\text { things not relevant to driving }\end{array}$ & 1 & 66 & -8 & 5 & -12 \\
\hline VEHO & Vehicles - opposite direction & -5 & -6 & 0 & 6 & 19 \\
\hline VEHS & Vehicles - same direction & 26 & 36 & 3 & -15 & 45 \\
\hline VEHP & Parked vehicle along right side & 69 & 0 & -12 & 2 & 2 \\
\hline NLANES & Number of lanes same direction & 3 & 3 & 0 & 7 & 41 \\
\hline TAREA & Area type & -70 & -20 & 15 & 2 & 6 \\
\hline LANDU & Land use & -26 & -19 & 11 & -24 & -18 \\
\hline LUMIN & Luminaires & 56 & 11 & 14 & 15 & 11 \\
\hline RSDET & $\begin{array}{l}\text { Road surface detail (right side } \\
\text { of road) }\end{array}$ & 55 & 22 & 2 & 41 & \\
\hline OTRSDET & Other than road detail & 60 & 61 & -6 & 25 & -4 \\
\hline
\end{tabular}

On the Road

$\begin{array}{llrrrr}\text { NPSON } & \text { Number of point sources } & 42 & 16 & -1 & -10 \\ \text { NMBRTON } & \text { Number of medium sized bright objects } & 69 & 16 & -4 & 13 \\ \text { NLBRTON } & \text { Number of large sized bright objects } & 58 & 4 & 22 & 18 \\ \text { NUALBON } & \begin{array}{l}\text { Number of units area of large sized } \\ \text { bright objects }\end{array} & 43 & -6 & 23 & 35 \\ & \end{array}$

\section{Area Left of Ellipse (excluding road)}

$\begin{array}{llcccc}\text { NPSLE } & \text { Number of point sources } & 10 & -7 & -1 & 3 \\ \text { NMBRTLE } & \text { Number of medium sized bright objects } & 32 & 48 & -12 & 10 \\ \text { NLBRTLE } & \text { Number of large sized bright objects } & 31 & 76 & -4 & 4 \\ \text { NUALBLE } & \text { Number of units area of large sized } & 37 & 67 & 4 & 10 \\ \end{array}$


Table 15. Variable factor weights (continued).

\begin{tabular}{|c|c|c|c|c|c|c|}
\hline $\begin{array}{c}\text { Variable } \\
\text { Name } \\
\end{array}$ & Description & $\begin{array}{c}\text { Factor } \\
1 \\
\end{array}$ & $\begin{array}{c}\text { Factor } \\
2 \\
\end{array}$ & $\begin{array}{c}\text { Factor } \\
3 \\
\end{array}$ & $\begin{array}{c}\text { Factor } \\
4 \\
\end{array}$ & $\begin{array}{c}\text { Factor } \\
5 \\
\end{array}$ \\
\hline & Area Right of $\mathrm{F}$ & Bllipse & & & & \\
\hline NPSRE & Number of point sources & 0 & 3 & 21 & 12 & 43 \\
\hline NMBRTRE & Number of medium sized bright objects & 16 & 37 & -6 & 37 & 22 \\
\hline NLBRTRE & Number of large sized bright objects & 0 & 74 & 3 & 20 & -12 \\
\hline NUALBRE & $\begin{array}{l}\text { Number of units area of large sized } \\
\text { bright objects }\end{array}$ & -3 & 68 & 7 & 20 & -20 \\
\hline
\end{tabular}

\section{Area in Ellipse}

$\begin{array}{llrrrr}\text { NPSIN } & \text { Number of point sources } & 8 & -1 & 13 & 22 \\ \text { NMBRTIN } & \text { Number of medium sized bright objects } & 2 & 34 & -1 & 32 \\ \text { NLBRTIN } & \text { Number of large sized bright objects }-17 & 12 & -5 & 16 & -28\end{array}$

\section{Variables Describing Area in the Ellipse}

\begin{tabular}{|c|c|c|c|c|c|}
\hline NTSIN & Number of traffic signs & -2 & 0 & 88 & -11 \\
\hline NTSWVRIN & $\begin{array}{l}\text { Number of traffic signs white/ } \\
\text { vertical rectangle }\end{array}$ & -7 & 4 & 89 & -11 \\
\hline EBRT & Recode of ellipse description & 25 & 40 & -4 & 62 \\
\hline EUN & Recode of uniformity & 5 & 5 & -13 & 55 \\
\hline
\end{tabular}

\section{Illuminance}

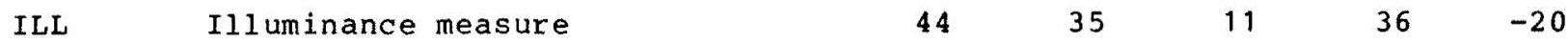

One Degree Radius of Target Center

\begin{tabular}{|c|c|c|c|c|c|c|}
\hline NPS 1 & Number of point sources & 4 & -26 & 0 & 28 & 25 \\
\hline NMBRT 1 & Number of medium bright sources & 0 & 3 & -3 & 49 & -4 \\
\hline NLBRT 1 & Number of large bright sources & -1 & 8 & -9 & 5 & -13 \\
\hline NTS 1 & Number of traffic signs & 5 & -4 & 85 & 1 & -2 \\
\hline NTSVR 1 & $\begin{array}{l}\text { Number of traffic signs - } \\
\text { vertical rectangle }\end{array}$ & -4 & 0 & 86 & -8 & 1 \\
\hline NTSHR 1 & $\begin{array}{l}\text { Number of traffic signs - } \\
\text { horizontal rectangle }\end{array}$ & 29 & -12 & -4 & -6 & 18 \\
\hline SURBRT 1 & Recode of surround brightness & -11 & 15 & -13 & 66 & I \\
\hline SURUN 1 & Recode of surround uniformity & 5 & 3 & 0 & 48 & \\
\hline
\end{tabular}


reliable procedure were available for estimating scene complexity. Eventually, such a procedure might be used to develop new sign luminance standards that would allow signs having reduced luminance in settings of low complexity.

This study is an attempt to develop a procedure for estimating the visual complexity of areas where signs might be placed. The study also seeks to determine if photographs of these locations can be used to evaluate their complexity, or if trips to the locations are necessary to view them from a vehicle.

\section{Definitions}

Many things influence the visibility of a sign, but one of the most important is the complexity of the sign location. So first, let's outline complexity. Imagine 2 signs of equal size and brightness. The first is mounted on the shoulder of a rural road that cuts through a forest, and you are driving on a dark night with your lights on high beam. Almost any sign will be visible under these conditions. Now imagine the second sign mounted at the corner of Broadway and 42 nd Street in New York City. Many things attract your attention: signs, lights, and detail all around you. In the city, the sign had better be very big and very bright, or it will be lost in the visual "noise."

Obviously these 2 cases are extremes, but background complexity doesn't have to be extreme to have an effect. Your task is to learn to accurately rate scene conditions -- to evaluate complexity.

Here are some terms we'll use:

- Scene - The "scene" is everything you see when you are looking at an area through the windshield of your car. It includes the road, the sky, and everything within your vision on both sides of the road. If you are looking at a photograph of a site, the scene is everything in the photograph.

- Cone - The "cone" is the portion of the right side of the road where you would normally look for traffic signs. It can extend from a few feet in front of your car to the horizon; but when driving, most people monitor an area in the cone from $200 \mathrm{ft}$ to $600 \mathrm{ft}(60$ $\mathrm{m}$ to $180 \mathrm{~m})$ down the road. The cone may include traffic signs, advertising signs, traffic lights, street lights, and even store fronts if they are near the road.

\section{Complexity Evaluation}

Skillful evaluation is a matter of asking yourself a series of questions systematically, and answering them based on what you observe. Consider the questions independently. Don't let a preceding question influence your response to one you consider later. Here are the questions and ways to answer them:

\section{How distracting is the scene?}

Drivers are less likely to see signs when they are distracted. A scene is distracting if it has medium or large bright lights and reflections to the left of the road or to the right of the cone. In the most distracting scenes, the cone may also have bright lights, bright reflections, and objects made visible by the lights. If something is dominant or unusual, it will add to the distraction. Although traffic signs along the road may themselves attract your attention, they should not be considered distracting. Traffic signs are considered in another question. Headlights of oncoming cars also should not be considered in this question.

Answer this question using the following scale:

Subjective Description Scale Definition

Very distracting

Distracting One or more things hold your attention

One or more things attract your attention

Average

One or more things may attract your attention

Not very distracting

Low potential for

Not disdistraction

tracting

Nothing distracts

\section{How many traffic signs are in the cone?}

Only the cone should be considered. Any traffic sign or object that looks like a traffic sign should be counted except signs you immediately identify as no-parking signs. If you are uncertain whether something is a traffic sign, it should be counted. If more than 2 signs appear on one pole, count the group as only 2 signs. 
Answer this question using the following scale:

\section{Subjective Description}

Five or more
Four
Three
Two
One or none

\section{How much detail is visible in the scene?}

Detail is anything you can see against the darkness. It includes lights and objects that the lights illuminate. Consider the entire field of view including the road, the horizon, and the area on both sides of the road. Can many objects be picked out? Is the scene cluttered with lights (including headlights) and reflections? Are there many pinpoint lights?

Answer this question using the following scale:

\section{Subjective} Description

A great deal of detail

$\begin{array}{ll}\begin{array}{l}\text { A lot of } \\ \text { detail }\end{array} & \begin{array}{l}60 \text { to } 808 \text { of the scene } \\ \text { is visible }\end{array} \\ \text { Average } & \begin{array}{l}40 \text { to } 608 \text { of the scene } \\ \text { is visible }\end{array} \\ \begin{array}{ll}20 \text { to } 408 \text { of the scene } \\ \text { is visible }\end{array} \\ \begin{array}{ll}\text { Very dittle } \\ \text { detail }\end{array} & \begin{array}{l}208 \text { or less of the scene } \\ \text { is visible (dark country } \\ \text { road) }\end{array}\end{array}$

\section{How many bright sources are in the scene?}

Are there many bright spots -streetlights, internaliy lighted signs, bright billboards, car lights, parking lot lights, lighted store windows, and bright reflections from glass and metal?

Answer this question using the following scale:

Subjective

Description

Very many

\section{Scale Definition}

The scene is saturated with bright sources (many

$\begin{array}{ll}\text { Many } & \text { Above average } \\ \text { Average } & \text { Moderate } \\ \text { Few } & \text { Some distant lights } \\ \text { Very few } & \begin{array}{l}\text { Virtually no bright } \\ \text { sources }\end{array}\end{array}$

\section{How much detail is visible in the cone?}

Are many lights and objects visible?

NOTE: Much detail may be visible in the scene, but very little in the cone. For example, a bridge approach may be lighted to the degree where you can see pavement seams. The horizon may be cluttered with lights. The cone, however, can include just a barely discernible bridge railing. Conversely, a dark country road with a lighted service station in the cone may exhibit little scene detail, but above average cone detail.

Answer this question using the following scale:

Subjective Description

A great deal of detail Scale Definition

808 or more of the cone is visible (city area with many lights and objects in the cone)

A lot of

60 to 808 of the cone is visible

Average

40 to $-60 \%$ of the cone is visible

Little detail 20 to 408 of the cone is visible

Very 1 ittle

$20 \%$ or less of the cone is visible (dark country road with virtually no illumination in the cone)

\section{How bright is the cone?}

The cone becomes bright as it fills with bright 1 ights and bright reflections. Signs are easier to recognize in a dark, uncluttered cone because contrast is increased and competition from the lights, reflections, and other detail is reduced. 
NOTE: Headlights of oncoming traffic can contribute many bright sources to the scene, but no brightness to the cone.

Answer this question using the following scale:

Subjective Description

Very bright

Bright

Mixed

Almost dark

Uniform ( $d a r k)$

108 or less of the cone is filled with $l$ ights and reflections

\section{How demanding would driving be at this location?}

Drivers' ability to detect and recognize traffic signs deteriorates as the demands of driving increase. The demands increase with the number of lanes and the number of vehicles traveling in the same direction. Many pinpoint lights to the left and right of the cone also increase the demands. Pedestrians and intersections controlled by traffic signals or signs add to the difficulty.

Answer this question using the following scale:

Subjective

Description

Very

demand ing

Demanding

Average

Not demanding
Easy

$$
\begin{aligned}
& 50 \% \text { or less of driver's } \\
& \text { time spent looking for } \\
& \text { driving information -- no } \\
& \text { problem lighting cigar- } \\
& \text { ette in this location }
\end{aligned}
$$

8. To be easily spotted, how bright would a new traffic sign have to be?

If a new traffic sign were placed in the cone approximately $500 \mathrm{ft}(150 \mathrm{~m})$ ahead, how bright would it have to be to successfully compete with its surroundings and be easily and consistently seen?

Answer this question using the following scale:

Subjective

Description

Scale Definition

Very bright

As bright as signs can be -- and even then it may be hard to pick out

Bright

Brighter than average

Average

Like most traffic signs

Not bright

Less than average brightness would be satisfactory

Dim

Almost any sign would be visible

\section{APPENDIX C. INSTRUCTIONS TO SUBJECT DRIVERS}

This study concerns the visibility of yellow diamond warning signs. You are going to drive a $24-\mathrm{mi}$ (38.4-km) loop around Trenton where a number of such signs have been placed along the right side of the road. Your task is to drive normally at or below the posted speed limit and watch for yellow diamond signs. We want to know when you first detect a yellow diamond sign and when you can read it. When you determine that a yellow diamond sign is visible, please say "detect" so we can record the location reading on a special odometer. When you are able to read the sign please do so aloud so the legibility distance can be recorded. Some yellow diamond signs contain symbols such as directional arrows or the silhouette of pedestrians. When these are encountered just say "symbol" when you determine that there are no words present.

We will provide instructions on the route to follow as you drive. Please stay at or below the posted speed limit, keep your headlights on low beam, stay in the rightmost lane as much as possible, and keep a considerable gap between your vehicle and traffic ahead of you. 
APPENDIX D SUGGESTED REVISION TO TRAFFIC CONTROL DEVICES HANDBOOK ${ }^{13}$

For warning signs only, where the visual complexity of the sign location is high and a single sign is being relied upon to transmit the information, it is suggested that one of the following sheetings be used:

- Type III sheeting.

- Type II sheeting, if replaced when it deteriorates to SIA 36 (72 percent of Federal specification).

Type III sheeting is also suggested when the sign is mounted on the left side of the road (no-passing pennants) or on severe right curves, because luminance decreases in these situations. Type II sheeting may be considered adequate for signs placed in situations of low visual complexity where the placement with respect to the roadway alignment does not demand Type III sheeting. A procedure for rating visual complexity is provided in a subsequent section.

\section{VISUAL COMPLEXITY}

Visual complexity refers to the characteristics of a sign location that make a sign more or less conspicuous. A procedure for rating visual complexity is provided below. The procedure, which applies only to warning signs mounted next to the right shoulder or roadway, is intended as a tool to improve sign maintenance management by directing limited resources to locations most in need of them. It is not intended to condone neglect of signs in less complex locations. Recording the visual complexity of a site in an automated sign inventory with other pertinent data will allow convenient use of this information.

One or 2 people should visit the site after dark and evaluate it at approximately 500 ft $(150 \mathrm{~m})$ from sign placement, or potential sign placement, using the 4 scales shown on the Visual Complexity Rating Form that follows. Evaluation of a site is a matter of asking the questions associated with each scale, and answering them based upon observation. The questions should be considered independently. A preceding question should not influence a response to a later one. After a response to each question is made, the corresponding scale value to the left should be circled. The scale values chosen are simply added, and the resulting number is the site's visual complexity value. For example, a site could be given the following values on the 4 complexity scales:

\begin{tabular}{rr} 
Scale 1 & 13 \\
Scale 2 & 8.5 \\
Scale 3 & -11.5 \\
Scale 4 & -1 \\
\hline & \\
Visual Complexity & 9
\end{tabular}

If the visual complexity value is greater than 11, the site is low complexity; when it is less than 9.5 , the site is high complexity.

The terms "scene" and "cone" used in the scale questions are defined below:

- Scene - Everything that can be seen when looking at a site through the windshield of a vehicle. It includes the road, the sky, and everything on both sides of the road.

- Cone - The portion of the roadway at the right where one would normally looks for traffic signs. It can extend from a few feet in front of the vehicle to the horizon, but commonly includes an area in the cone from $200 \mathrm{ft}$ to $600 \mathrm{ft}(60 \mathrm{~m}$ to 180 m) ahead of the vehicle.

13 The first paragraph is written to be inserted in the Traffic Control Devices Handbook (U.S. Department of Transportation, Federal Highway Administration, 1983) section on Sign Reflectorization, page 2-27. The rest is written as a new section, Visual Complexity, to be inserted between the sections on Sign Reflectorization and Il lumination. 


\section{How much detail is visible in the scene?}

Detail is anything you can see against the darkness. It includes lights and objects that the lights illuminate. Consider the entire field of view including the road, the horizon, and the area on both sides of the road. Can many objects be picked out?

Answer this question by circling the appropriate scale value:

\begin{tabular}{|c|c|c|}
\hline Scale Value & $\begin{array}{l}\text { Subjective } \\
\text { Description }\end{array}$ & Scale Definition \\
\hline 25 & $\begin{array}{l}\text { A great deal } \\
\text { of detail }\end{array}$ & 808 or more of the scene has visible detail \\
\hline 21 & $\begin{array}{l}\text { A lot of } \\
\text { detail }\end{array}$ & 60 to 808 of the scene has visible detail \\
\hline 17 & Average & 40 to 608 of the scene has visible detail \\
\hline 13 & Little detail & 20 to 408 of the scene has visible detail \\
\hline 9 & $\begin{array}{l}\text { Very little } \\
\text { detail }\end{array}$ & $\begin{array}{l}208 \text { or less of the scene has visible detail (dark } \\
\text { country road) }\end{array}$ \\
\hline
\end{tabular}

\section{How many bright sources are in the scene?}

Are there many bright spots -- streetlights, internally lighted signs, bright billboards, car lights, parking lot lights, lighted store windows, and bright reflections from glass and metal?

Answer this question by circling the appropriate scale value:

Scale Value $\quad \begin{aligned} & \text { Subjective } \\ & \text { Description }\end{aligned}$

$22.5 \quad$ Very many

$\begin{array}{ll}19 & \text { Many } \\ 15.5 & \text { Average } \\ 12 & \text { Few } \\ 8.5 & \text { Very few }\end{array}$

\section{Scale Definition}

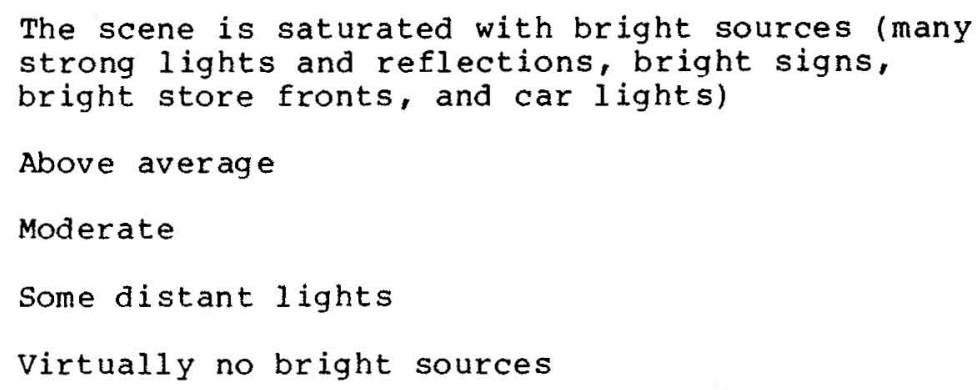

Virtually no bright sources 


\section{How much detail is visible in the cone?}

Are many lights and objects visible?

NOTE: Much detail may be visible in the scene, but very little in the cone. For example, a bridge approach may be lighted to the degree where you can see pavement seams. The horizon may be cluttered with lights. The cone, however, can include just a barely discernible bridge railing. Conversely, a dark country road with a lighted service station in the cone may exhibit little scene detail, but above average cone detail.

Answer this question by circling the appropriate scale value:

$\begin{array}{cl}\text { Scale Value } & \begin{array}{l}\text { Subjective } \\ \text { Description }\end{array} \\ -22.5 & \begin{array}{l}\text { A great deal } \\ \text { of detail }\end{array} \\ -17 & \text { A lot of detail } \\ -11.5 & \text { Average } \\ -6 & \text { Little detail } \\ -0.5 & \begin{array}{l}\text { Very little } \\ \text { detail }\end{array}\end{array}$

\section{Scale Definition}

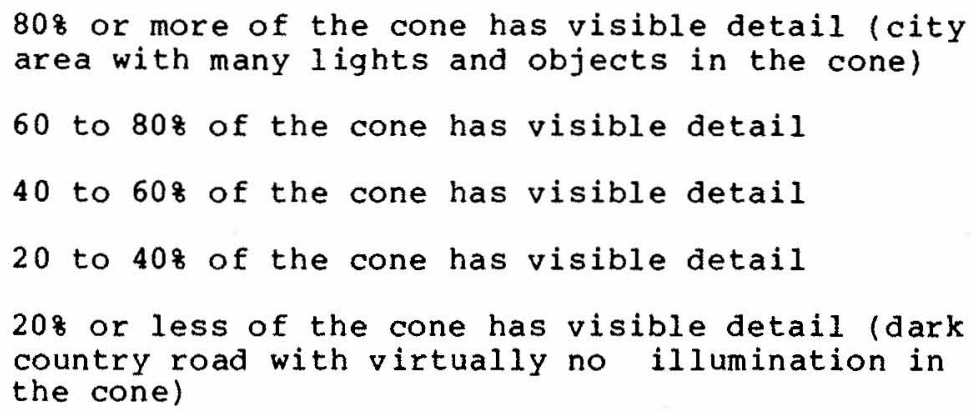

\section{How demanding would driving be at this location?}

Drivers' ability to detect and recognize traffic signs deteriorates as the demands of driving increase. The demands increase with the number of lanes and the number of vehicles traveling in the same direction. Many pinpoint lights to the left and right of the cone also increase the demands. Pedestrians and intersections controlled by traffic signals or signs add to the difficulty.

Answer this question by circling the appropriate scale value:

Scale Value

$-25$

- 19 Demanding

- 13 Average

- 7 Not demanding

- 1 Easy
Subjective Description

Very demanding

\section{Scale Definition}

80 \& or more of driver's time spent looking for driving information -- would not try to light cigarette in this location

70 to $80 \%$ of driver's time spent looking for driving information

60 to 708 of driver's time spent looking for driving information

50 to 608 of driver's time spend looking for driving information

$50 \%$ or less of driver's time spent looking for driving information -- no problem lighting cigarette in this location 
Baker, C. A., Morris, D. F., \& Steedman, W. C. Target recognition on complex displays. Human Factors, 1960, 2 (2), 51-61.

Banks, W. P., Bachrack, K. M., \& Larson, D. W. The asymmetry of lateral interference in visual filter identification. Perception and Psychophysics, 1977, 22, $232-240$.

Banks, W. P., Bodinger, D., \& Illige, M. Visual detection accuracy and target-noise proximity. Bulletin of the Psychonomic Society, 1974, 2, 411-414.

Bloomfield, J. R. Visual search in complex fields: size differences between target disc and surrounding discs. Human Factors, 1972, 14, 139-148.

Brown, B., \& Monk, T. H. The effect of local target surround and whole background constraint on visual search times. Human Factors, 1975, 17, 81-88, 357-360.

Cahill, M. C., \& Carter, R. C. Color code size for search displays on different density. Human Factors, 1976, 18, 273-280.

Cole, B. L., \& Jenkins, S. E. The nature and measurement of conspicuity. Proceedings of 10 th Australian Road Research Board Conc., University of Sydney, 1980, 10, part 4, 99-107.

Crawford, A. The perception of light signals: the effect of the number of irrelevant lights. Ergonomics, 1962, 5, 417-428.

Dornic, S., \& Borg, G. Visual search for simple geometric figures: the effect of target-noise similarity. Reports from the Institute of Applied Psychology, 1971, 22, Stockholm, Sweden, University of Stockholm.

Eriksen, C. W. Object location in a complex perceptual field. Journal of Experinental Psychology, 1953, 45, 126-132.

Eriksen, B. A., \& Eriksen, C. W. Effects of noise letters upon the identification of a target letter in a nonsearch task. Perception and Psychophysics, 1974, 16, 143149 .

Estes, W. R., \& Wolford, G. L. Effects of spaces on report of tachistoscopically presented letter strings. Psychonomic Science, 1971, 25, 77-80.

Ezekiel, M., \& Fox, K. A. Methods of correlation and regression analysis (3rd ed.). New York: John Wiley and Sons, Inc., 1963.

Forbes, T. W., Fry, Jr., J. P., Joyce, R. P., \& Pain, R. F. Letter and sign contrast, brightness, and size effects on visibility. Highway Research Record, 1968 , $216,48-54$.

Forbes, T. W., Pain, R. F., Fry, Jr., J. P., \& Joyce, R. P. Effect of sign position and brightness on seeing simulated highway signs. Highway Research Record, 1967, $164,29-37$.

Forbes, T. W., Pain, R. F., Joyce, R. P., \& Fry, Jr., J. P. Color and brightness factors in simulated and full-scale traffic sign visibility. Highway Research Record, $1968,216,55-65$.

Green, B. F., \& Anderson, L. K. Color coding in visual search task. Journal of Experimental Psychology, 1956, 51(1), 19-24.

Hanson, D. R., \& Dickson, A. D. Significant visual properties of some fluorescent pigments. Highway Research Record, 1963, 49, 13-29.

Lovie, A. D., Lovie, P. The effect of mixed visual contrast schedules on detection times for both free and horizontally structured visual search. Ergonomics, 1970, $13,735-741$.

Mace, D. J., Perchonok, K., \& Pollack, L. Traffic signs in complex visual environments (FHWA-RD-82-102). Washington, DC: U. S. Department of Transportation, Federal Highway Administration, November 1982. 
Mace, D. J., \& Pollack, L. Visual complexity and sign brightness in detection and recognition of traffic signs. Transportation Research Record, 1983, 904, 33-41.

Mackworth, N. H. Visual noise causes tunnel vision. Psychonomic Science, 1965, 3 , 67-68.

McIntyre, C., Fox, R., \& Neale, J. Effects of noise similarity and redundancy on the information processed from brief visual displays. Perception and Psychophysics, $1970,7,328-332$.

McGee, H. W., Moore, W., Knapp, B. G. \& \& Sanders, J. H. Decision sight distance for highway design and traffic control reguirements (FHWA-RD-78-78). Washington, DC: U.S. Department of Transportation, Federal Highway Administration, February 1978.

McNemar, Q. Psychological statistics (3rd ed.). New York: John Wiley and Sons, Inc., 1963.

Monk, T. H. Target uncertainty in applied visual search. Human Factors, 1976, 18, $607-612$.

Pain, R. Brightness and brightness ratio as factors in the attention value of highway signs. Highway Research Record, 1969, 275, 32-40.

Perchonok, K., \& Pollack, L. Luminance requirements for traffic signs, a comparison of sign performance and requirements (FHWA-RD-81-158). Washington, DC: U.S. Department of Transportation, Federal Highway Administration, December 1981.

Saenz, N. E., \& Riche, C. B. Shape and color as dimensions of a visual redundant code. Human Factors, $1974,16,308-313$.

Shaw, P. Processing of tachistoscope displays with controlled order of characters and spaces. Perception and Psychophysics, 1969, 6, 257-266.

Smith, S. L. Color coding and visual search. Journal of Experimental Psychology, $1962,64,434-440$.

Smith, S. L., \& Thomas, D. W. Color versus shape coding in information displays. Journal of Applied Psychology, 1964, 48, 137-146.

Steedman, W. C., \& Baker, C. A. Target size and visual recognition. Human Factors, $1960, \underline{2}, 120-127$.

U.S. Department of Transportation, Federal Highway Administration. Standard alphabets for highway signs. Washington, DC: Author, 1972.

U.S. Department of Transportation, Federal Highway Administration. Standard specifications for construction of roads and bridges on federal highway projects, FP-79, Section 633. Washington, DC: Author, 1979.

U.S. Department of Transportation, Federal Highway Administration. Traffic control devices handbook. Washington, DC: Author, 1983, 2-27.

Wolford, G. L., \& Hollingsworth, S. Retinal location and string position as important variables in visual information processing. Perception and psychophysics, $1974,16,437-442$.

NOTES

1. Jenkins, S. E. Consideration of the effects of background on sign conspicuity. Draft paper accepted with minor revisions for 11 th Conference of the Australian Road Research Board. 


\section{FEDERALLY COORDINATED PROGRAM (FCP) OF HIGHWAY RESEARCH, DEVELOPMENT, AND TECHNOLOGY}

The Offices of Research, Development, and Technology (RD\&T) of the Federal Highway Administration (FHWA) are responsible for a broad research, development, and technology transfer program. This program is accomplished using numerous methods of funding and management. The efforts include work done in-house by RD\&T staff, contracts using administrative funds, and a Federal-aid program conducted by or through State highway or transportation agencies, which include the Highway Planning and Research (HP\&R) program, the National Cooperative Highway Research Program (NCHRP) managed by the Transportation Research Board, and the one-half of one percent training program conducted by the National Highway Institute.

The FCP is a carefully selected group of projects, separated into broad categories, formulated to use research, development, and technology transfer resources to obtain solutions to urgent national highway problems.

The diagonal double stripe on the cover of this report represents a highway. It is color-coded to identify the FCP category to which the report's subject pertains. A red stripe indicates category 1, dark blue for category 2 , light blue for category 3 , brown for category 4, gray for category 5 , and green for category 9 .

\section{FCP Category Descriptions}

\section{Highway Design and Operation for Safety}

Safety RD\&T addresses problems associated with the responsibilities of the FHWA under the Highway Safety Act. It includes investigation of appropriate design standards, roadside hardware, traffic control devices, and collection or analysis of physical and scientific data for the formulation of improved safety regulations to better protect all motorists, bicycles, and pedestrians.

2. Traffic Control and Management

Traffic RD\&T is concerned with increasing the operational efficiency of existing highways by advancing technology and balancing the demand-capacity relationship through traffic management techniques such as bus and carpool preferential treatment, coordinated signal timing, motorist information, and rerouting of traffic.

\section{Highway Operations}

This category addresses preserving the Nation's highways, natural resources, and community attributes. It includes activities in physical maintenance, traffic services for maintenance zoning, management of human resources and equipment, and identification of highway elements that affect the quality of the human environment. The goals of projects within this category are to maximize operational efficiency and safety to the traveling public while conserving resources and reducing adverse highway and traffic impacts through protections and enhancement of environmental features.

\section{Pavement Design, Construction, and Management}

Pavement RD\&T is concerned with pavement design and rehabilititation methods and procedures, construction technology, recycled highway materials, improved pavement binders, and improved pavement management. The goals will emphasize improvements to highway performance over the network's life cycle, thus extending maintenance-free operation and maximizing benefits. Specific areas of effort will include material characterizations, pavement damage predictions, methods to minimize local pavement defects, quality control specifications, long-term pavement monitoring, and life cycle cost analyses.

\section{Structural Design and Hydraulics}

Structural RD\&T is concerned with furthering the latest technological advances in structural and hydraulic designs, fabrication processes, and construction techniques to provide safe, efficient highway structures at reasonable costs. This category deals with bridge superstructures, earth structures, foundations, culverts, river mechanics, and hydraulics. In addition, it includes material aspects of structures (metal and concrete) along with their protection from corrosive or degrading environments.

\section{RD\&T Management and Coordination}

Activities in this category include fundamental work for new concepts and system characterization before the investigation reaches a point where it is incorporated within other categories of the FCP. Concepts on the feasibility of new technology for highway safety are included in this category. RD\&T reports not within other FCP projects will be published as Category 9 projects. 
FHWA/RD-85/056/9-85(150)QE

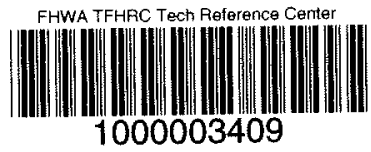

